\title{
Machine learning and deep learning techniques to support the clinical diagnosis of arboviral diseases: A systematic review
}

\section{Sebastião Rogério da Silva Neto}

Universidade de Pernambuco

Thomás Tabosa Oliveira

Universidade de Pernambuco

Igor Vitor Teixeira

Universidade de Pernambuco

Samuel Benjamin Aguiar de Oliveira

Fundação de Medicina Tropical Dr. Heitor Vieira Dourado

Vanderson Souza Sampaio

Fundação de Medicina Tropical Dr. Heitor Vieira Dourado

Theo Lynn

Dublin City University

Patricia Takako Endo ( $\nabla$ patriciaendo@gmail.com )

Universidade de Pernambuco https://orcid.org/0000-0002-9163-5583

\section{Systematic Review}

Keywords: Arboviruses, Dengue, Chikungunya, Zika, Clinical diagnosis, Machine Learning, Deep Learning, Systematic Review, Backward Snowballing

Posted Date: August 11th, 2021

DOl: https://doi.org/10.21203/rs.3.rs-799039/v1

License: (c) (1) This work is licensed under a Creative Commons Attribution 4.0 International License. Read Full License 


\title{
Machine learning and deep learning techniques to support the clinical diagnosis of arboviral diseases: A systematic review
}

Sebastião Rogério da Silva Neto ${ }^{1}$, Thomás Tabosa Oliveira ${ }^{1}$, Igor Vitor Teixeira ${ }^{1}$, Samuel Benjamin Aguiar de Oliveira ${ }^{2,3}$, Vanderson Souza Sampaio ${ }^{2,3}$, Theo Lynn ${ }^{4}$, Patricia Takako Endo ${ }^{1, *}$

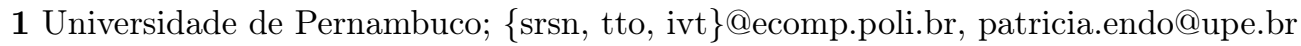
2 Universidade do Estado do Amazonas; \{sbadoliveira, vandersons\}@gmail.com 3 Fundação de Medicina Tropical Dr. Heitor Vieira Dourado

4 Dublin City University; theo.lynn@dcu.ie

* Correspondence: patricia.endo@upe.br

\begin{abstract}
Background: Neglected tropical diseases (NTDs) primarily affect the poorest populations, often living in remote, rural areas, urban slums or conflict zones. Arboviruses are a significant NTD category spread by mosquitoes. Dengue, Chikungunya, and Zika are three arboviruses that affect a large proportion of the population in Latin and South America. The clinical diagnosis of these arboviral diseases is a difficult task due to the concurrent circulation of several arboviruses which present similar symptoms, inaccurate serologic tests resulting from cross-reaction and co-infection with other arboviruses. Objective: The goal of this paper is to present evidence on the state of the art of studies investigating the automatic classification of arboviral diseases to support clinical diagnosis based on Machine Learning (ML) and Deep Learning (DL) models. Method: We carried out a Systematic Literature Review (SLR) in which Google Scholar was searched to identify key papers on the topic. From an initial 963 records (956 from string-based search and 7 from single backward snowballing technique), only 15 relevant papers were identified. Results: Results show that current research is focused on the binary classification of Dengue, primarily using Tree based ML algorithms and only one paper was identified using DL. Five papers presented solutions for multi-class problems, covering Dengue (and its levels) and Chikungunya. No papers were identified that investigated models to differentiate between Dengue, Chikungunya, and Zika. Conclusions: The use of an efficient clinical decision support system for arboviral diseases can improve the quality of the entire clinical process, thus increasing the accuracy of the diagnosis and the associated treatment. It should help physicians in their decision-making process and, consequently, improve the use of resources and the patient's quality of life.
\end{abstract}

\section{Introduction}

Neglected tropical diseases (NTDs) include a wide range of parasitic, viral, and bacterial diseases that prevail in tropical and subtropical conditions in 149 countries and affect one billion people every year [1. One major category of NTDs are arthropod-borne viruses (or arbovirus diseases), a group of viruses that are found in nature and biologically transmitted between susceptible vertebrate hosts by hematophagous arthropods [2]. 
Arboviruses included a wide variety of diseases including African swine fever virus, Japanese encephalitis virus, Rift Valley fever virus, tick-borne encephalitis virus, West Nile virus or yellow fever virus however the most common are Dengue, Chikungunya and Zika [3]. These three arboviruses are primarily transmitted by Aedes spp. mosquitoes, of which Aedes aegypti and Aedes albopictus are the most common vectors [4.5]. The Aedes aegypti can easily adapt to urban and semi-urban areas [6.7]. Population growth, unplanned urbanization, habitat modification, human and animal migration, and climate change, combined with low-quality housing and neglected peri-domestic environments, all contribute to creating ideal ecological conditions for urban Aedes spp. populations to thrive $[5,8,10]$. These factors, disproportionately affecting the poor, increase the geographical area at risk to arboviral diseases and contribute to establishment of arboviruses as a global health problem 8-11. Furthermore, these arboviruses are maintained outside rainy seasons by transovarial transmission from female mosquitoes to offspring [12,13]. Other modes of transmission include vertical and sexual transmission [14 15], and contaminated transfusions [5, 16]. The overall burden of arboviral diseases in general is significant. The incidence and number of deaths due to Dengue are increasing resulting in a global burden of disease of 2.9 million disability-adjusted life years (DALYs) for 2017 alone, a 107\% increase since 1990 [17. Recent analysis on the global burden of Chikungunya and Zika suggest an average yearly loss of over 106,000 and 44,000 DALYs, respectively, between 2010 and 2019 [18. In each case, the burden of these diseases disproportionately impacts the Americas.

While the clinical presentation of these diseases are well-established [19,20], diagnosing these diseases is a difficult task. Three primary reasons are cited in the literature to explain why there are difficulties to make an arboviral diagnosis. Firstly, the majority of cases are asymptomatic thus arbovirus may be present in an area without an identifiable outbreak 21,22. Secondly, their symptomatic infection is usually clinically indistinguishable from each other. All of them share common symptoms like fever, arthralgia, myalgia, headache, retro-orbital pain 21. While Dengue and Zika have some distinct symptoms, for example, hemorrhagic diathesis (Dengue) and edema in limbs (Zika), and Chikungunya is related to the prominent joint complaint, their diagnosis requires a high degree of experience and clinical insight which can be further complicated in special populations 22, 23. In addition, Dengue and Chikungunya symptoms may include hemorrages and leukopenia/thrombocytopenia, while Chikungunya and Zika symptoms may include non-purulent conjunctivitis [22]. Thirdly, co-infection is also common increasing the difficulty of diagnosis of these conditions [5, 21, 24, 25].

Despite the difficulties in differential diagnosis, the progression and impact of these diseases varies significantly. After infection by Dengue, the disease may manifest asymptomatically and patients may not even know they are infected. Serologically, after 7-10 days after the mosquito bite, a diagnosis of Dengue can be confirmed [26]; some people may experience symptoms such as fever, headache, pain in the muscles and joints, and fatigue. For some, the disease may progress to a more severe condition resulting in bleeding, organ damage, and plasma leakage [19]. Dengue can be classified into two stages - the febrile phase and the critical phase. The febrile phase typically lasts for 2-7 days. The critical phase of dengue begins at defervescence and typically lasts $24-48$ hours. While most patients clinically improve, some may experience systemic vascular leakage syndrome, characterised by increasing hemoconcentration, hypoproteinemia, pleural effusion, and ascites [27. Severe Dengue can result in death due to plasma leaking, fluid accumulation, respiratory distress, severe bleeding, or organ impairment [19]. Chikungunya infection may manifest symptoms similar to Dengue between the fourth and seventh day after the bite, but with greater joint pain. The progression of Chikungunya has three phases. The acute phase is characterised with 
sudden onset symptoms manifesting with high fever, rash, and arthralgia, affecting mainly the small and large joints. The subacute phase is characterised by worsening arthralgia. While Chikungunya is rarely fatal, it can progress to a chronic state. Post-Chikungunya rheumatism is common and can last from weeks years with associated adverse effects on quality of life [5.28 31]. Zika was initially considered a mild disease sometimes with no fever episode [5], however it is now clear that its major threat is related to microcephaly and other congenital abnormalities in the fetus and newborn; it may trigger Guillain-Barré syndrome, neuropathy, and myelitis in adults and older children 31. Symptoms of Zika include arthralgia, edema of the extremities, low fever, maculopapular rash that is often pruritic, headaches, retro-orbital pain, without purulent conjunctivitis, vertigo, myalgia, and digestive disorder [32]. The most serious manifestation of infection is Congenital Zika Syndrome (CZS). The risk of the infection can occur during any gestational trimester [33]. CZS is related to fetal microcephaly, fetal brain disruption sequence, subcortical calcifications, pyramidal, and extrapyramidal signs, ocular abnormalities (focal pigmented mottling, chorioretinal atrophy), congenital contractures, fetal growth restriction, and even death [33 35].

Early identification of specific arbovirus infections can have a significant impact on the clinical course and decisions related to treatment and care. The adverse impact of poor arbovirus diagnosis are exacerbated where there are competing pressures for funding and trained and experienced staff, due to multiple concurrent disease epidemics 36 . Novel low-cost scalable approaches to the differential diagnosis of arboviral diseases for epidemiological surveillance are required. One such approach is the development of computational models for monitoring and diagnostic classification based on clinical data and symptoms. Machine Learning (ML) and Deep Learning (DL) models have been widely proposed in biomedical field to support the diagnosis and prediction of disease 37]. ML is a computational method that makes use of experience to make predictions, i.e., it is an algorithm that receives input data (training data set) to learn or find a pattern. Data quality and size are fundamental to the success of the learning process and as consequence to guarantee the efficiency of the model predictions. When designing a ML model, the goal is to find a configuration (a set of hyperparameters) that produces a model able to generalise and produce a satisfactory performance when dealing with previously unseen new data. DL is a sub-field of ML which emphasises learning based on successive layers of increasingly meaningful representations [38. Here, "deep" is related to the idea of successive layers of representations. DL models are based on early iterations of neural networks and are increasingly reported as the most effective ML approach with the advantage of combining the feature extraction and the classification task at the same time. However, the "black box" nature of most DL models is a significant challenge in the health space which values transparency. As such more transparent ML models are commonly used due their interpretability.

In this paper, we detail a Systematic Literature Review (SLR) on how existing research employs ML and DL techniques to automatically classify arboviral diseases and support clinical diagnosis.

\section{Methods}

The purpose of an SLR is to identify, select and critically appraise research on a specific topic. SLRs typically comprise three main phases: planning the review, conducting the review, and reporting the review results [39. The goal of this paper is to present evidence on the state of the art of studies investigating the automatic classification of arboviral diseases to support clinical diagnosis based on ML and DL models. To accomplish this goal, this SLR seeks to address the following research questions: 
- RQ 01: What arboviruses are the focus of research on ML and DL classification of arboviral diseases to support clinical diagnosis?

- RQ 02: Which ML and DL techniques are being used in research relating to the classification of arboviral diseases to support clinical diagnosis?

- RQ 03: How are ML and DL models being designed and how do they perform when classifying arboviral diseases?

- RQ 04: What data characteristics are considered when applying the ML and DL techniques?

- RQ 05: What are the metrics being used to evaluate the performance of the ML and DL techniques?

\subsection{Search Strategy}

The search strategy comprised an automated and manual phase. A literature search was conducted using Google Scholar with the following search string: (("deep learning" OR "machine learning") AND ("arbovirus" OR "arboviral") AND ("classification" OR "diagnosis" OR "analysis") AND ("clinical data")), in March, 2021. We then performed a manual search to identify and download relevant studies from a single iteration reverse snowballing procedure applied to relevant studies found in the automated search.

\subsection{Study Selection}

To ensure the selection of studies relevant to our review, we consider studies that meet specific inclusion criteria. Inclusion criteria required that papers be in the English language, and make use of clinical data in the application of ML or DL models for arbovirus diagnosis in a primary study. Papers were excluded if they (1) did not examine the diagnosis of arboviruses, (2) were in a language other than English, (3) were a secondary or tertiary study, (4) used conventional statistical techniques, or (5) did not use clinical data as inputs to the ML and DL models.

The initial automated search returned 956 records. These had their title and abstracts assessed by two independent authors according to the inclusion and exclusion criteria. Where a conflict arose, a third author arbitrated on selection. Based on the on the inclusion and exclusion criteria, nine papers were retained. We performed a single backward snowballing, that returned seven papers, and it yielded a further six relevant papers, resulting in 15 papers.

\subsection{Data Extraction and Coding}

The following data was extracted for each study: authors, publication year, arboviral disease type(s), ML and DL technique(s) employed, the data set used in the study, the data characteristics used as input, and metrics used to evaluate the ML and DL

performance.

\section{Results and discussions}

Appendix A presents a list of included studies by year of publication, classification target, ML and/or DL techniques used, model configurations, software, evaluation metrics, and optimisation techniques employed for hyperparameter and feature selection. 


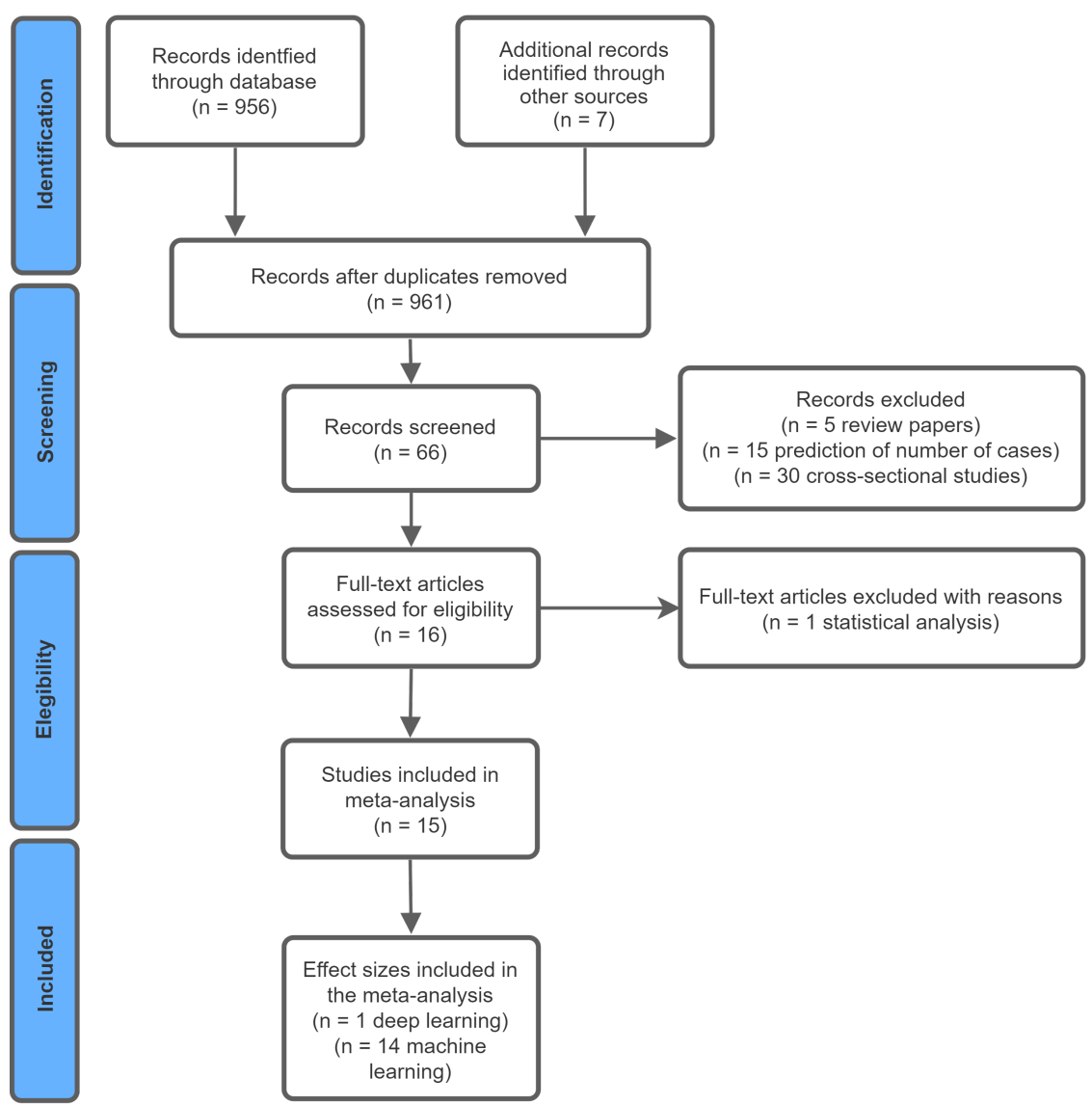

Figure 1. Study selection.

\subsection{What arboviruses are the focus of research on Machine Learning and Deep Learning classification of arboviral diseases to support clinical diagnosis?}

Surprisingly, given the range of arboviral diseases, the focus or research, albeit a small sample, was the three most popular diseases i.e. Dengue, Chikungunya, and Zika. Although these three are common arboviral diseases, no studies were found that carried out multi-classification considering these arboviral diseases or other arboviral types, such as West Nile virus, yellow fever virus, Saint Louis Encephalitis virus, Mayaro virus, Oropouche virus and others, showing that there are space for further investigations in this area.

Most of works presented models for binary classification: Dengue or not [40 45]; DHF or not [46]; Chikungunya or not [47]; and Zika classified between "Discarded cases" and "Somewhat probable" for CZS [48]. It is interesting to note that the only work that deals with Zika is focused on CZS, not covering Zika in general.

And four studies covered multi-class problem: Thitiprayoonwongse et al. [49] classified between Dengue Fever (DF), Dengue Hemorrhagic Fever 1 (DHF1), Dengue Hemorrhagic Fever 2 (DHF2) and Dengue Hemorrhagic Fever 3 (DHF3); Fahmi et al. [50 focused only on Dengue, classifying between DF, Dengue Hemorrhagic Fever (DHF) and DSS; Veiga et al [48] that classified between "Discarded cases", "Somewhat probable", "Moderately probable" and "Highly probable" of having CZS, while Lee et al. 51 proposed models to differentiate between DF, DHF and Chikungunya. 


\subsection{Which Machine Learning and Deep Learning techniques are being used in research relating to the classification of arboviral diseases to support clinical diagnosis?}

Figure 2 presents the ML and DL techniques used to perform arboviral diseases classification in the sampl $]^{1}$. Only one paper used DL. Ho et al. [45 compared a Convolutional Neural Network (CNN) with Decision Tree and Logistic Regression models. All other papers employ common ML techniques, including tree-based algorithms (Decision Trees, Random Forest, AdaBoost and Gradient Boost), Neural Networks (NN), Support Vector Machine (SVM), Naive Bayes, Logistic Regression, and K-Nearest Neighbors (kNN).

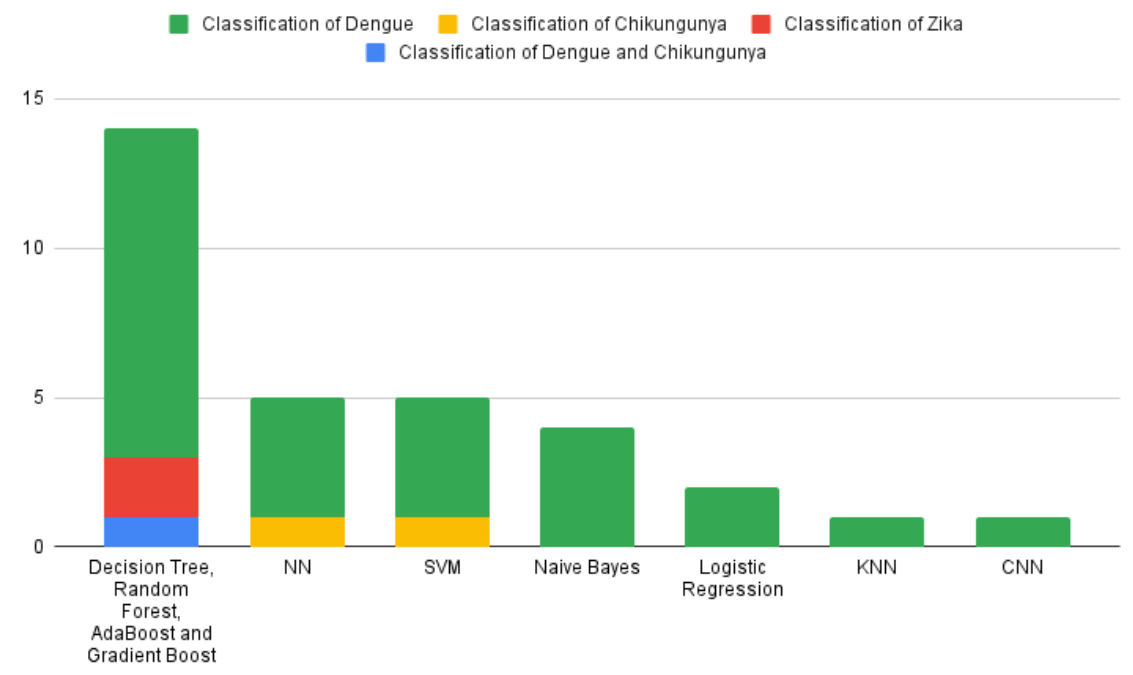

Figure 2. Models used in the works divided by the main problems.

\subsubsection{Tree based algorithms: Decision Tree, Random Forest, AdaBoost and Gradient Boost}

A Decision Tree is a non-parametric method that can be applied in problems with categorical variables (classification tree, the focus of this work) and also with continuous variables (regression tree). A tree is composed of a root node, internal nodes and leaf nodes, and it is built successively dividing data according to one of the predictor variables [53]. To build a Decision Tree, it is necessary to define the node-splitting algorithm, in order to minimise the impurity of the node. If the split achieves the maximum reduction of impurity, then the node is defined as a leaf [54. The most common splitting algorithms are the Information Gain (used by Classification and Regression Tree (CART)) and the Gini index (used by Iterative Dichotomiser 3 (ID3) and C4.5 algorithms). The main advantage of using Decision Tree algorithms is implicit feature selection during the model building process and the interpretability of results. Decision Trees are also able to handle missing values, which are commonly encountered in clinical studies [40]. On the other hand, over-complex trees do not generalise the data well, often presenting overfitting (or underfitting), and are prone to errors with

\footnotetext{
${ }^{1}$ For the purpose of this paper, we consider traditional statistical techniques (e.g. Decision Trees, Logistic Regression and Naive Bayes) as ML as per [52.
} 
relatively small number of samples for training. The sample includes eleven Decision Tree models - 440], [55], [49], [51], [56], [42], [43], [44], [45], [50] and [48].

In contrast to Decision Trees (sometimes referred to as 'strong learner' approaches) optimised to solve a specific problem by looking for the best possible solution, ensemble learning techniques are based on a set of "weak learners". Ensemble learning techniques can be categorised into three classes: (1) bagging (or bootstrapping), (2) boosting and (3) stacking [57]. Random Forest is an ensemble technique based on bagging that combines several Decision Trees. It is built randomly from a set of possible trees with $K$ characteristics in each node. Random in this context means that in the set of trees, each tree has an equal chance of being sampled. Multiple classification trees are obtained from bootstrap samples in order to calculate the final majority classification. The SLR sample includes three Random Forest models - [46], [50] and [48. As Random Forest models combine different Decision Trees, their results are not as easy to understand as a Decision Tree and are also more expensive computationally. Notwithstanding this, Random Forests typically outperform Decision Trees and handle balancing errors better when working with an imbalanced data set [58.

Boosting is an ensemble technique that combines $k$ low performance models $(M 1, M 2 \ldots, M k)$ in order to improve the final model, $M *$ [59]. The $k$ classifiers are learned iteratively and after a $M i$ is learned, the weights are updated in order to generate the next classifier, $M i+1$. Performance is improved by training tuples that were misclassified by $M i$. The final boosted model, $M *$, combines the results of each $k$ classifier.

The Adaptive Boosting (AdaBoost) $\sqrt[60]{6}$ is the first stepping stone in boosting techniques and it uses Decision Trees with single split (one node and two leaves), also named Decision Stumps, as "weak learners". Gradient Boost uses a technique named forward stage-wise additive modelling that add a new Decision Tree at each step to minimise a global cost function using the Steepest Gradient Descent method [57]. The main advantages of boosting algorithms in general, including AdaBoost and Gradient Boost, are intrinsic automated variable selection, and flexibility regarding the type of predictors and stability when handling high-dimensional data 61]. AdaBoost, particularly, is also known to be quite resistant to overfitting. While these advantages have attracted attention of biomedical researchers 61], only one paper in the sample proposed an AdaBoost model (Fahmi et al. [50]) and another, Veiga et al. [48], proposed a Gradient Boost model.

\subsubsection{Support Vector Machine (SVM)}

SVM is a classifier based on Vapnik's statistical learning theory 62 . To perform classification, SVM builds hyperplanes in a multidimensional space in order to separate instances of different classes. The goal is to find the optimal separating hyperplane and, at same time, maximise the distance between the support vectors (which are the extreme delimiters) 57,62 .

Robustness is one of the main advantages of SVM models. Data with outliers do not impact negatively in SVM model performance. While Decision Tree models benefit from interpretability, lack of transparency is a drawback of SVM models understand, especially when dealing with high-dimensional data sets. SVM models can also be quite memory-intensive and therefore processing large and complex data sets can be slow [57]. SVM models feature in five studies in the sample - [41, [46], 44, [47] and [50].

\subsubsection{Neural Networks}

MLP 63 is a perceptron model that simulates a human neuron. Its main difference from a simple perceptron is the number of hidden layers, each containing a number of 
neurons which are connected by weights. The data of the independent variables is inserted in the neurons of the input layer, is processed in the hidden layers, and finally, the output layer is responsible for presenting the result of the MLP. Sometimes, MLPs are often referred to as NN and the terms are used interchangeably. In general, NNs present many advantages including high capacity to learn and generalise, and the ability to deal with imprecise, fuzzy, noisy and probabilistic information 64 65. As such, they are widely used in health research 66 68.

MLP was a popular ML solution in the 1980s with applications in various fields, recently the interest in this type of model was renewed due to the success of DL. Several authors classify MLP as a traditional model of ML 69,70, but with the advent of DL, concepts of MLP were improved and it can also be classified as a DL 71. In this paper, we classify MLP as a traditional model of ML due to the context observed in the selected proposals. Five papers employed NN models in the sample: [72, [42], [43], 447] and [50].

\subsubsection{Naive Bayes}

Naive Bayes is a probabilistic classifier that performs classification based on the Bayes' Theorem, selecting the most likely class according to its independent variables 73 . The term naive is due to the way the model calculates the probabilities of each event i.e. all attributes of the data set are equally important and independent.

In general, Naive Bayes models are simple, fast and effective, and perform well when a data set contains outliers or is missing data [57, common features in many health data sets. However, Naive Bayes models are not without drawbacks. They assume that all attributes of a data set have the same importance which is often not true. If a data set has large numbers of attributes, the reliability of the results may be limited. Four works in the sample applied Naive Bayes models - [41, [43], [46] and [50].

\subsubsection{Logistic Regression}

Logistic Regression is a classification technique based on idea of modelling the odds of belonging to Class 1 using an exponential function [57. In this technique, the dependent variable, $Y$, is binary and the independent variables, $X=\left\{x_{1}, x_{2}, \ldots, x_{n}\right\}$, are used to estimate the value of $Y$ by using a logistic function. The goal is to find an optimal hyperplane, that separates the two target classes (binary classification). In case of multi-class problems, the one-vs-all strategy can be used to address the problem.

Some advantages of this model includes dealing with categorical independent variables and a high degree of reliability. As disadvantages, this type of model does not generalise well when using a large number of features, it is vulnerable to overfitting, and cannot solve non-linear problems, requiring a transformation of non-linear resources [74]. Two works employed Logistic Regression in the sample - [45] and [50].

\subsection{6 k-Nearest Neighbours (kNN)}

$\mathrm{kNN}$ is a classification technique that defines a minimum number of neighbours, $k$, and calculates the distance, the similarity, of each data element with respect to its $k$ neighbours. There are many measures of similarity, and the most commons approaches are the Euclidean distance and the Minkowski metric [57]. The most frequent class among the neighbouring $k$ is determined as the class of the target instance 75 .

Simplicity is the main advantage of $\mathrm{kNN}$. $\mathrm{kNN}$ is a good choice when working with a small low-dimensional data set however it can be extremely inefficient when dealing with large data sets because it is computes all pairwise distances [57]. Only one paper in the sample proposed a kNN model, Fahmi et al. [50], using Euclidean distance with $k=5$. 


\subsubsection{Convolutional Neural Networks (CNN)}

A convolutional neural network is a DL technique. CNNs are designed to process input data in the form of multiple arrays $[76]$. A basic CNN comprises convolutional layers, pooling layers, nonlinear function (generally ReLU), and fully connected layers. Units in a convolutional layer are organised into feature maps and each unit is connected to local patches in the feature maps of the previous layer. It is done by using a set of weights called filters. The result of this local weighted sum is passed through a nonlinear activation function. All units in a feature map share the same filter bank, and different feature maps in one layer can use different filter banks. The result of this whole process feeds fully connected layers resulting in a final classification. As discussed earlier, DL models, including CNN, often outperform traditional ML models however their adoption in health settings has suffered due to lack of transparency.

Although the methodology of training and test the models is clearly well-defined, the resultant models themselves can be often unexplainable to humans 77 . Even when techniques are used to select attributes resulting in good model performance, the relationships between those attributes and the output classification may not directly track causal relationships in the real world 77]. One paper in the sample, Ho et al. 45, uses a CNN, DenseNet. DenseNet is a CNN architecture in which each layer is connected to all the others within a dense block 78 . In this case, all layers can access feature maps from their preceding layers enabling heavy feature reuse. As a direct consequence, the model is more compact and less prone to overfitting. Furthermore, each individual layer receives direct supervision from the loss function through the shortcut paths, which provides implicit deep supervision 79 .

\subsection{How are ML and DL models being designed and how do they perform when classifying arboviral diseases?}

As presented in Section 3.1, studies included in the sample focused on only three arboviruses - Dengue, Chikungunya, and Zika.

\subsubsection{Dengue}

Of the 15 relevant papers in the sample, 12 included the diagnosis of Dengue in their studies including binary classification and multi-class classification:

\section{- Binary classification}

- Dengue or not Dengue: Tanner et al. [40, Fathima and Hundewale [41], Sajana et al. [42], Gambhir et al. [43], Sanjudevi and Savitha [44], Ho et al. 45;

- Severity of Dengue: Tanner et al. $402^{2}$, Potts et al. [55, Phakhounthong et al. [56;

- Risk of Dengue: Faisal et al. 72]; and

- DHF or not: Arafiyah et al. 46

- Multi-class classification

- DF, DHF or Dengue Shock Syndrome (DSS): Fahmi et al. [50]; and

- DHF1, DHF2 or DHF3: Thitiprayoonwongse et al. 49

\footnotetext{
${ }^{2}$ Tanner et al. appeared twice because they presented two distinct classification problems.
} 
Tanner et al. [40 proposed two Decision Tree models. The first, the Dengue Diagnostic Model (DDM), sought to classify if a patient had Dengue or not using 1,200 records of patients with acute febrile illness. The second, the Dengue Severity Prediction Model (DSPM), sought to classify the severity of Dengue in adults using data from 161 patients. A C4.5 Decision Tree classifier was built using the Inforsense software. A $\mathrm{k}$-fold cross-validation approach $(k=10)$ was used to avoid model over-fitting. Both models presented good performance however the DDM performed better across all metrics i.e. sensitivity $(71.2 \%)$, specificity $(90.1 \%)$, overall error rate $(15.7 \%)$ and AUC (0.88). This is unsurprising given the larger data set available to the DDM.

Fathima and Hundewale [41] compared two classification models, SVM and Naive Bayes, to classify if a patient had Dengue or not. To determine the best SVM hyperparameters, a Grid Search was performed changing the gamma parameter and the cost (c). Despite executing the Grid Search, neither the best configuration nor the configuration of the Naive Bayes model was detailed. In general, the SVM model presented the best performance, despite its low sensitivity (47\%). The Naive Bayes models presented high sensitivity and very low specificity, accuracy and risk rates, all above of $18 \%$.

Sajana et al. [42 proposed three models for binary classification of Dengue using clinical and laboratory data: a MLP, and two Decision Trees (C4.5 and CART). Like Fathima and Hundewale [41], the configurations of the models were not detailed. The CART model presented the best results, achieving $100 \%$ in all metrics (accuracy, sensitivity, precision and F-Measure). There is no mention regarding the use of feature selection and hyperparameter optimisation. Given the results, it is possible that the models were overfitting due to the small amount of data available, only 20 records (see Table 1).

Gambhir et al. 43] proposed three models (NN, Decision Tree and Naive Bayes) to classify whether a patient had Dengue or not. The configuration of the models were described in the paper and are summarised in Table 5 K-fold cross-validation $(k=10)$ was used to validate and test the models. The NN presented the best results $-79.09 \%$ accuracy, $55.55 \%$ sensitivity, and $88.5 \%$ specificity. However, the other models achieved similar performance. Gambhir et al. 43 did not describe whether hyperparameter optimisation or feature selection was applied in the data set.

Sanjudevi and Savitha 44 compared Decision Tree and SVM models to classify whether a patient had Dengue or not. Model configurations were not described. The WEKA tool was employed to run the experiments and calculate the metrics. The SVM model obtained the best results achieving $100 \%$ sensitivity, $100 \%$ specificity, $100 \%$ precision and an $99 \%$ AUC. The extremely high performance results suggest model overfitting.

Ho et al. 45 proposed three models to classify Dengue using clinical data - a Decision Tree, a Logistic Regression, and a CNN. Models were validated and tested using $\mathrm{k}$-fold cross-validation $(k=10)$. Feature selection was performed using crude odds ratio and adjusted odds ratio analysis. From 18 available attributes, four were initially selected - age, body temperature, white blood cell counts and platelets counts. An additional three experiments were performed with more attributes: (1) six attributes, the four previously mentioned plus gender and haemoglobin count; (2) 11 attributes, the previous six and five more vital signals; and (3) the entire data set with 18 attributes. Results suggested that when using only four attributes, the AUC in all experiments were close to $84 \%$; the CNN performed marginally better than Decision Tree and Logistic Regression models.

Potts et al. 55] proposed Decision Trees to classify pediatric patients into "severe" or "non-severe" Dengue cases. The stopping rules used to create the trees were described in the paper and are summarised in Table 5 . Five scenarios were evaluated with different 
definitions of Severe Dengue: (1) Tree 1 considered Severe Dengue as DSS, and used four attributes from 11 (WBC, HTC, MOMO\%, PLT); and (2) Tree 2 defined Severe Dengue as DHF Grade 3 or 4 or pleural effusion index $(P E I)>15$, and used five attributes (Age, WBC, PLT, NEUT\%, AST); (3) Tree 3 defined Severe Dengue as DSS or required intravenous fluid; (4)Tree 4 defined Severe Dengue as DSS or PLT less than 50,000; and (5) Tree 5 defined Severe Dengue as DSS or received fluid intervention.

Trees 3, 4 and 5 were not described in the work because, according to Potts et al. [55], they did not obtain any significant improvement in relation to Trees 1 and 2. Both Decision Trees 1 and 2 have the same initial splitting variable, WBC, reinforcing the utility of this variable in distinguishing Severe Dengue. Models were validated and tested using $\mathrm{k}$-fold cross-validation $(k=5)$ and result $\mathrm{3}^{3}$ suggested that Trees 1 and 2 achieved a sensitivity metric in excess of $90 \%$ for the "Severe" class, while the sensitivity for the "non-Severe" class was below $50 \%$.

Phhakhounthong et al. 56 proposed a CART Decision Tree model to classify Dengue severity based on clinical and laboratory attributes. They performed a Logistic Regression analysis to determine the significance of each attribute to compose the tree. In their case, the most significant factor in predicting severe dengue was haematocrit. The results obtained from k-fold cross-validation $(k=10)$ for binary classification of Severe Dengue were $60.5 \%$ sensitivity, $65 \%$ specificity, and $64.1 \%$ accuracy.

Phhakhounthong et al. [56] state that tree pruning and tuning parameters were applied to optimise the model but did not describe the settings used for the experiment.

Despite having performed a feature selection with Logistic Regression, the results did not exceed $65 \%$ in the metrics evaluated.

Faisal et al. [72 sought a binary classification of Dengue risk, differentiating patients as "high risk" or "low risk". An MLP model was proposed for the classification task, and a Grid Search technique was performed to optimise the model configuration, changing four parameters: number of neurons, momentum, learning rate, and number of iterations) Seven attributes were selected using Self Organizing Map (SOM) and the model achieved $70 \%$ accuracy.

Thitiprayoonwongse et al. 49 classified a patient as DF, DHF1, DHF2 and DHF3 using a Decision Tree. Two data sets were used, one from the Srinagarindra Hospital and another from the Songklanagarind Hospital. Three experiments were performed: (1) using only data from the Srinagarindra Hospital; (2) using only Songklanagarind Hospital data; and (3) using data from both hospitals. The attributes of both two data sets were not described, but the attributes selected to compose the Decision Tree for each experiment were presented. In Experiment (1), six attributes were selected: shock, leakage, bleeding, platelet, liver size and je-vaccine. In Experiment (2), nine attributes were selected: shock, leakage, bleeding, platelet, abdominal pain, rash, uri, hematocrit, AST. In Experiment (3) eight attributes were selected: shock, leakage, bleeding, platelet, ALT, lymp, WBC (count and minimal count). The configuration of the Decision Tree was changed only in the degree of confidence parameter in Experiments (1) and (2); no detail was provided for Experiment (3). Experiment (1) presented the best overall results. It is interesting that even with the addition of one more data set, Experiment (3) largely did not achieve superior results. The class posing the greatest classification challenge, DHF1, reported the lowest values in all three experiments although all were greater than $80 \%$.

Arafiyah et al. [46 proposed and evaluated three models to classify DHF or not DHF - Random Forest, SVM and Naive Bayes. Unfortunately, there is no information about the model configurations. The models were trained and the metrics were

\footnotetext{
${ }^{3}$ The work did not explicitly present any metrics for evaluating the models, such as accuracy or sensitivity. However, in the results, a table was presented with values that can be interpreted as the sensitivity of the "Severe" and "non-Severe" classes.

${ }^{4}$ It is noteworthy that in the Grid Search technique process, each attribute was tested individually.
} 
calculated using the Orange tool. Random Forest achieved better results than SVM and Naive Bayes models: $79.6 \%$ accuracy, $84.1 \%$ precision, $82.2 \%$ sensitivity, $83.1 \%$ F1-Score and $89.8 \%$ AUC. No details on hyperparameter optimisation or feature selection were provided.

Fahmi et al. [50] evaluated eight models for classifying Dengue into three categories: DF, DHF and DSS. The models included NN, SVM, kNN, Decision Tree, Random Forest, Naive Bayes, AdaBoost, and Logistic Regression. The configuration of all models were described and are summarised in Table 5 Experiments were carried out in two different scenarios: (1) without feature selection, and (2) with feature selection using the ReliefF techniqu 5 . In both scenarios, the best result was obtained by the NN model with $71.3 \%$ accuracy, $70.8 \%$ precision, and $71.3 \%$ sensitivity in Scenario (1) and with $72 \%$ accuracy, $71.5 \%$ precision, and $72 \%$ sensitivity in Scenario (2). Results showed that the feature selection did provide significant improvements.

In summary, those studies in our sample addressing the diagnosis of Dengue primarily focused on binary classification (11); only two studies performed multi-class classification. Multi-class classification studies sought to Dengue subtypes [49] or different levels of disease severity [50. The prevalence of binary classification reflects its simpler nature. Multi-class classification is both more complex to perform and interpret, and consequently results are often inferior to simpler models. This is reflected in our survey [49,50]. Tree based models (Decision Tree and derivatives) were the most common technique used in Dengue classification (10); nine of which used simple Decision Trees, often obtaining better results than other benchmark models. It is important to highlight that despite tree-based learning algorithms being broadly used for classification problems due their simplicity for implementation and interpretability of results, the usage of imbalanced data sets can skew the performance of such models, exacerbating inadequacies inherent in the tree splitting criterion 81. It was noted that a number of studies likely suffered model overfitting [42,44 however further analysis is not possible due to the lack of detail in their publications, although it should be noted that each of these studies used the smallest data sets (see Section 3.4. The usage of different data sets and lack of detail regarding both model configuration, feature selection, and hyperparameter optimisations made comparisons of these studies difficult. For example, six of the 12 studies $41,42,44,46,51,56$ did not present any description of their proposed models thus adversely impacting future reproducibility.

\subsubsection{Chikungunya}

Only one study in the sample sought to classify Chikungunya. Hossain et al. [47] proposed a Specialized Belief Rules System (BRBES) to classify Chikungunya using clinical data containing vital signs and symptoms, and considering severity classes as output (very high, high, medium and low). The BRBES system was compared with a NN, an SVM and a Fuzzy Logic Based Expert System (FLBES) as well as expert opinions. Due to the scope of our work, we consider only the ML models for analysis, NN and SVM. The NN outperformed the SVM model, obtaining an AUC of $81.1 \%$ vs $80.8 \%$. Notwithstanding the small difference in performance between these two ML models, neither models outperformed the BRBES system. A significant limitation of this study is the lack of detail on model configurations thereby adversely impacting comparability and reproducibility.

\footnotetext{
${ }^{5}$ ReliefF is an algorithm developed by Kira and Rendell in 1992 80 that takes a filter-method approach to feature selection that is notably sensitive to feature interactions.
} 


\subsubsection{Zika}

In relation to the Zika classification, only one study was identified. Veiga et al. 48] sought to classify suspected cases of CZS using clinical and non-clinical data. The authors compared five algorithms: kNN, CART Trees, Random Forest, AdaBoost and Gradient Boost. After performing a Grid Search, only two of the candidate models were selected and described in the final publication - (1) the Random Forest model was used with a data set without textual data to address a binary classification ("Discarded cases" and "Somewhat probable"); and (2) the Gradient Boost model was used with a data set with supplementary textual data to handle a multi-class output ("Discarded cases", "Somewhat probable", "Moderately probable" and "Highly probable"). For the binary classification, the Random Forest model obtained 91\% sensitivity and and F1-Score of $83 \%$ for the "Discarded cases" class however exhibited significantly poorer results for the "Somewhat probable" class with 50\% sensitivity and an F1-Score of $61 \%$, . During the execution of Grid Search, the tree-based models obtained a similar performance and all were superior to kNN. The small but better performance of Random Forest in relation to other tree-based models is probably due to its bootstrapping process that helps to avoid overfitting when using small data sets as per Group 1 (272 samples).

For the multi-class problem, the Gradient Boost model presented good performance mainly for the "Discarded cases" class with $91 \%$ in all metrics (precision, sensitivity and F1-score). As the amount of data used in this experiment is greater (1109 samples) than the binary classification, the Gradient Boost obtained a better performance. However, Veiga et al. 48 did not provide details on the proportion of data in each class. As such, it is not possible to analyse whether any data imbalance impacted the models performance. This is the only study where the code of the final models are available for download 6

\subsubsection{Differential Arboviral Diagnosis}

Given the difficulties in differential diagnosis of arboviruses discussed in Section 1], it was surprising that only one study was identified that sought to distinguish between two different arboviral diseases, in this case Dengue and Chikungunya. Lee et al. [51] proposed models to differentiate between DF, DHF and Chikungunya cases. Four experiments were presented - (1) DF and Chikungunya using only clinical data; (2) DF and Chikungunya using clinical and laboratory data; (3) DHF and Chikungunya using clinical data only; and (4) DHF and Chikungunya using clinical and laboratory data. For each classification, a Decision Tree model was developed using $\mathrm{R}$ software. Details on model configuration were not provided. Results suggested that Decision Trees using clinical and laboratory data outperformed the models using only clinical data.

\subsubsection{Cross-validation}

It is worth noting that a number of studies used cross-validation techniques to validate and test their models. [40, [43, , 45, 56] [50] and [48 used k-fold cross-validation with $k=10$; and $[55$ also used $\mathrm{k}$-fold but with $k=5$. Lee et al. [51] was the only study that applied the leave-one-out (LOO) cross-validation. Commonly, cross-validations are recommended when handling with small data sets, and in an attempt to minimise the learning bias. LOO cross-validation is a type of $\mathrm{k}$-fold cross-validation in which $k$ is the number of samples in the data set. Therefore, despite taking advantage of each data point, LOO cross-validation can be computationally expensive, especially if the data set is large. Note that Lee et al. had a data set composed of 1,034 samples however they

\footnotetext{
${ }^{6}$ https://github.com/rafael-veiga/Classification-algorithm-of-Congenital-Zika-Syndromecharacterizations-diagnosis-and-validation
} 
did not mention anything about the computational effort needed to execute the experiments.

\subsection{What attributes are considered when applying the Machine Learning and Deep Learning techniques?}

\subsubsection{Summary of Data Sets and Attributes}

Table 1 summarises the data sets used by the included studies in this SLR by number of records, number of attributes, input for models, period of the data, and location. While the number of attributes presented in this table were described as available in a focal data set, in some cases, the studies did not use all of them for training and testing their proposed models (see Sub-section 3.4.2.

Table 1. Characteristics of the data sets used to evaluate ML and DL models for arboviral diseases classification

\begin{tabular}{|c|c|c|c|c|c|}
\hline Classification & Records & Attributes & Input for models & Period & Location \\
\hline \multicolumn{6}{|l|}{ Dengue } \\
\hline Tanner et al. 40] & 1,200 & 41 & 3 and 5 & Not described & Singapore and Vietnam \\
\hline Fathima and Hundewale 41 & 5,000 & 29 & Not described & Not described & India \\
\hline Sajana et al. 42 & 20 & 12 & 12 & Not described & India \\
\hline Gambhir et al. 43 & 110 & 16 & 16 & 2015 to 2016 & India \\
\hline Sanjudevi and Savitha 44 & 108 & 17 & Not described & Not described & Not described \\
\hline Ho et al. $\overline{45}$ & 4,894 & 18 & $4,6,11$ and 18 & 2015 & Taiwan \\
\hline Potts et al. 55 & 1,230 & 11 & 11 & $1994-97,1999-2002,2004-07$ & Thailand \\
\hline Phakhounthong et al. 56 & 1,180 & 23 & 5 & 2009 to 2010 & Cambodja \\
\hline Faisal et al. $\overline{72}$ & 210 & 40 & 7 & Not described & Not described \\
\hline Thitiprayoonwongse et al. $\overline{49}$ & 1001 & 400 & 6,8 , and 9 & Not described & Thailand \\
\hline Arafiyah et al. & 213 & 4 & 4 & 2005 & Indonesia \\
\hline $\begin{array}{l}\text { Fahmi et al. } \overline{50} \\
\text { Chikungunya }\end{array}$ & 14,019 & 16 & 10 & 2016 to 2019 & Indonesia \\
\hline $\begin{array}{r}\text { Hossain et al. } \\
\text { Zika }\end{array}$ & 250 & 5 & 5 & Not described & Bangladesh \\
\hline Veiga et al. 48] & 1,501 & 13 & 7 & 2015 & Brazil \\
\hline \multicolumn{6}{|l|}{ Dengue and Chikungunya } \\
\hline Lee et al. 51] & 1,034 & 33 & 5 & 2004 and 2008 & Singapore \\
\hline
\end{tabular}

Tanner et al. [40 used a data set composed of 1,200 patient records from Singapore and Vietnam with acute febrile illness. The data set is composed of 15 clinical (symptoms and vital signs) and 26 laboratory attributes. The selected attributes for the Dengue classification model were platelet count, white blood cells and lymphocyte count, body temperature, haematocrit count, and neutrophil count. For the classification of Dengue severity, the selected attributes were platelet count, the crossover value $(\mathrm{Ct})$ of the real-time RT-PCR for dengue viral RNA, and the presence of anti-dengue IgG antibodies.

Fathima and Hundewale 41] used a data set comprising 5,000 records of patients with Dengue from Chennai and Tirunelveli, India. The data set includes details on 29 patient symptoms. The structure of the data set is not provided in sufficient detail to infer the extent to which the data set is balanced or unbalanced; most of the data would appear to be related to non-Dengue patients.

Sajana et al. 42 used the data collected from various medical wards of hospitals in Vijayawada, India; it comprises only 20 records with 12 attributes. We highlight that the joint/muscle pain attribute used by Sajana et al. [42] was referenced in Table 3 as joint swelling and myalgia. As no feature selection technique is referenced in the paper, we assumed all attributes were used for model training.

Gambhir et al. 43 used clinical and non-clinical data acquired from patients in Delhi from 2015 and 2016. The data set contains 110 records - 85 positive Dengue cases and 25 negative Dengue cases. Each record has 16 attributes, of which nine are clinical data (age, gender, vomit, abdomen pain, chills, bodyache, headache, weakness, and 
fever) and the remainder physical examination/laboratory data (temperature, heart rate, platelet count, dengue antigen NS1 or serology $(\operatorname{IgM}, \operatorname{IgG}) 7^{7}$.

Sanjudevi and Savitha [44 used a data set composed of 108 records with 17 attributes. The attributes were not detailed in the paper and no feature selection technique was performed.

Ho et al. 45] used data from the National University Hospital Cheng Kung (NCKUH) in Tainan City, Taiwan. The data set comprised 4,894 records of clinical and laboratory data including 2,942 cases of laboratory-confirmed Dengue cases and 1,952 non-Dengue cases. Ho et al. 45 analysed odds ratios to select four attributes and create a subset of data; two additional subsets were created with six and 11 attributes based on the initial subset. In the experiments, the data set with all attributes was also used for comparison purposes however there was no evidence that more attributes contributed to improved model performance.

Potts et al. 55] used data from 1,384 pediatric patients with Dengue and DHF, with 11 attributes. After initial screening, 1,230 records were included in the analysis - 208 cases of DHF, 374 of DF, and 648 of Other febrile illness (OFI). Data were collected in Thailand for the periods 1994 to 1997, 1999 to 2002, and 2004 to 2007.

Phakhounthong et al. [56] used a data set comprising 1,225 records related to febrile episodes in children from Angkor Hospital for Children, Cambodia. From those 1,225 records, 198 were confirmed cases of Dengue; only 38 were Severe Dengue cases. The data set included information about demographic, clinical and laboratory data. Logistic Regression was used and these five attributes were selected for model training.

Faisal et al. 72 used a data set with records of 210 patients with 40 attributes, divided into demographic, clinical, laboratory and Bioelectrical Impedance Analysis (BIA) parameters measurements to classify risk of Dengue. Laboratory data were used to classify baseline patient risk and thus create the output attribute for model training. This procedure was performed using an unsupervised model, SOM. After that, another SOM model was used to perform feature selection to define the attributes to be used as input for the proposed model; ultimately seven attributes were selected.

Thitiprayoonwongse et al. [49] used two data sets: one composed of information from 524 patients from Srinagarindra Hospital, Thailand and another with 477 patients from Songklanagarind Hospital, Thailand to classify DF, DHF1, DHF2 or DHF3. The Decision Tree model selected different attributes for each experiment.

Arafiyah et al. [46] used a medical data set comprising 213 records using only four clinical data sources - temperature, presence of spotting, presence of bleeding, and tornikuet test. The complete list of the attributes present in the data set was not described by the authors, so there is no way to know if or how feature selection was applied.

Fahmi et al. [50 used a data set that was provided by the Disease Prevention and Control Division in Central Java, Indonesia to classify DF, DHF or DSS. After the verification of the missing values, selection of relevant attributes, and data normalisation, the final data set comprised 14,019 records with 16 attributes including demographic, epidemiological, clinical and laboratory (hematological) information. Despite having 16 attributes available, after the application of the feature selection procedure, only ten attributes remained for model training and testing based on their importance.

As discussed, only one study addressed Chikungunya classification. Hossain et al. trained and tested their model using a data set comprising 250 records collected from various hospitals in Dhaka and Chittagong, Bangladesh. The data set had five attributes indicative of patient symptoms i.e. fever, muscle pain, joint pain, headache

\footnotetext{
${ }^{7}$ In the original work, these data were mistakenly classified. Gambhir et al. 43 considered age, gender, vomit, abdomen pain, chills, bodyache, headache, weakness, and fever as non-clinical data; and platelet count, temperature, heart rate, dengue antigen NS1, IgM, IgG, dengue NS1 antigen as clinical.
} 
and swelling in the joints, each classified as high, medium or low intensity.

In relation to Zika classification, Veiga et al. 48 sought to classify suspected cases of CZS using clinical and non-clinical data. A data set with 1,501 records of live newborns suspected of microcephaly reported in the Public Health Event Registry (RESP) and the National Birth Registration System (SINASC) from Brazil was considered. This data set contains information about demographic, epidemiological, clinical (signs), laboratory (serological and others). From 13 attributes, seven were used as input for the model. Additionally, there is also textual data provided by the health professional when registering the newborns' information in the system such as reports, descriptions and other possible observations. Veiga et al. [48] separated the records into two groups where Group 1 contained only clinical and non-clinical data (272 records), and Group 2 containing clinical, non-clinical and complementary textual notes (1,109 records). The most frequent terms presented in the notes were used to assist the classification. Group 2, which considered these textual notes, obtained better results compared to Group 1.

Lee et al. 51] used demographic, epidemiological, clinical and laboratory data with 1,034 records to train and test their model to distinguish between two different arboviral diseases: Dengue and Chikungunya. While 36 attributes are identified in the study, only five were used for training and testing the model i.e. period of symptoms, fever, fever (duration), bleeding and platelet count. Of the 1,034 records, 917 were related to adult Dengue patients confirmed by PCR test, including 55 records related to DHF. 117 were records related to Chikungunya patients confirmed by RT-PCR. The Chikungunya data were collected in August 2008, while the Dengue data were collected during the large 2004 Dengue outbreak, both in Singapore.

It is important to note that none of the included studies explicitly describe or discuss how they handle imbalanced data (between-class imbalance). Given how the data sets were reported in the papers, none of the models were trained with a data set with similar number of records per class (see Table 2. The data set used by Tanner et al. [40] presents this imbalancing issue in which the DSS class represents only 0.016 of the entire data set while the non-DF class presents 0.696. A similar situation is found in the data set used by Lee et al. [51] in which DHF is 0.053 and DF is 0.833. Although not explicitly mentioned, Lee at al. [51 applied the LOO cross-validation, as described in sub-section 3.3.4, that can be considered an alternative when evaluating models under imbalanced data sets. According to He et al. 81], when presented with complex imbalanced data sets, most standard learning algorithms "fail to properly represent the distributive characteristics of the data and resultantly provide unfavorable accuracies across the classes of the data". It happens essentially because ML models learn by reducing the error and do not take into consideration the class proportion. In health, where the minority class is commonly the positive case for the target disease (or the rare case), it is desirable that a classifier provides high accuracy for the minority class, without severely impacting on the performance of the majority class 81 . Three exceptions were identified in our sample. Gambhir et al. [43] and Ho et al. 45] used data sets in which the number of Dengue cases is larger than non-Dengue; and Fahmi et al. [50] used a large data set, in which there were more DHF cases than DF.

Additionally, the combination of imbalanced data and small sample size issue was also found in this SLR. For example, Gambhir et al. [43 used a data set composed of 110 records with 85 positive cases of Dengue and 25 negative cases. Other works also presented a small data set, such as $42,44,46,47,72$, having less than 250 records each, however none of them described the distribution of the classes. As stated by He et al. [81], in this case, "traditional learning algorithms often fails to generalise inductive rules over the sample space" and when samples are limited, the rules formed can become too specific, leading to overfitting. This is likely to be the case in Sajana et al. [42] and Sanjudevi and Savitha [44. To address these issues, some methods, such as sampling, 
Table 2. Distribution of samples per classes.

\begin{tabular}{|c|c|c|c|}
\hline & Classes & Samples & Proportion* \\
\hline \multicolumn{4}{|l|}{ Dengue } \\
\hline \multirow{4}{*}{ Tanner et al. 40} & Non-DF & 836 & 0.696 \\
\hline & DF & 173 & 0.144 \\
\hline & $\mathrm{DHF}$ & 171 & 0.142 \\
\hline & DSS & 20 & 0.016 \\
\hline \multirow[t]{2}{*}{ Gambhir et al. 43} & Non-Dengue & 25 & 0.227 \\
\hline & Dengue & 85 & 0.772 \\
\hline \multirow[t]{2}{*}{ Ho et al. 45} & Non-Dengue & 1,952 & 0.398 \\
\hline & Dengue & 2,942 & 0.601 \\
\hline \multirow[t]{3}{*}{ Potts et al. 55} & OFI & 648 & 0.526 \\
\hline & DF & 374 & 0.304 \\
\hline & DHF & 208 & 0.169 \\
\hline \multirow[t]{2}{*}{ Phakhounthong et al. 56$]^{* *}$} & Dengue & 160 & 0.808 \\
\hline & Severe Dengue & 38 & 0.191 \\
\hline \multirow[t]{4}{*}{ Thitiprayoonwongse et al. 49} & $\mathrm{DF}$ & 488 & 0.487 \\
\hline & DHF 1 & 222 & 0.221 \\
\hline & DHF 2 & 229 & 0.228 \\
\hline & DHF 3 & 62 & 0.061 \\
\hline \multirow[t]{3}{*}{ Fahmi et al. 50} & $\mathrm{DF}$ & 4,870 & 0.347 \\
\hline & $\mathrm{DHF}$ & 8,540 & 0.609 \\
\hline & DSS & 609 & 0.043 \\
\hline \multicolumn{4}{|l|}{ Dengue and Chikungunya } \\
\hline \multirow[t]{3}{*}{ Lee et al. 51} & DF & 862 & 0.833 \\
\hline & $\mathrm{DFH}$ & 55 & 0.053 \\
\hline & Chikungunya & 117 & 0.113 \\
\hline
\end{tabular}

${ }^{*}$ Numbers were rounded. ${ }^{* *}$ There are 982 samples of non-Dengue cases in this data set with an overall total of 1,180 records as shown in Table 1 but as this class was not considered in the problem, we did not use it for calculating the proportion.

cost-sensitivity, kernel-based and active learning [81] are available in the literature.

\subsubsection{Attributes of the data sets}

Figure 3 presents the types of attributes found in the data sets described previously. Demographic, epidemiological and clinical (symptoms, signs and co-morbidities) data were grouped as resource-limited attributes following the terminology presented by Lee et al. [51]; specific equipment is not specified for these data as they were collected at the time of the appointment. Laboratory attributes (hematological, biochemical and serological) and others are grouped as well-resourced attributes because they require specific equipment to be performed.
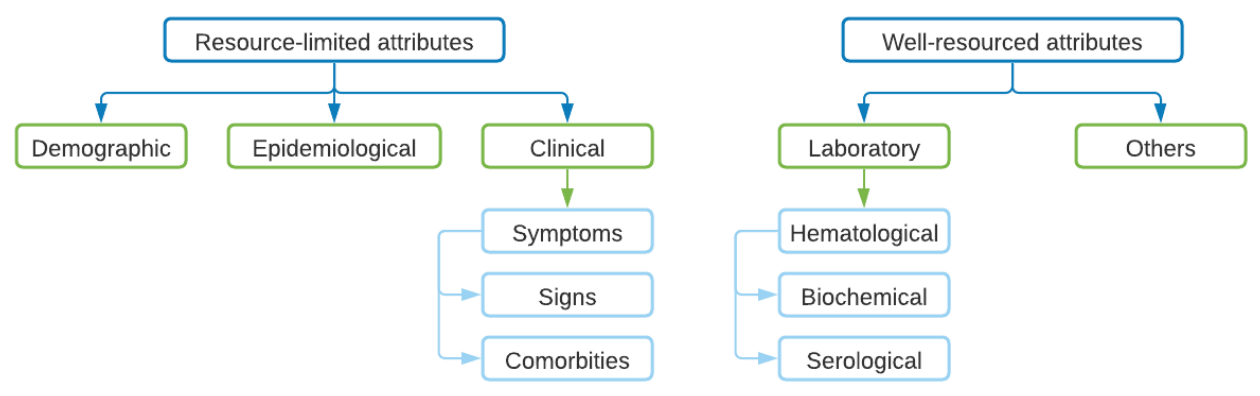

Figure 3. Attributes found in the data sets.

Table 3 presents a summary of all demographic, epidemiological and clinical data present in the data set used by the 15 included studies. Despite the focus on studies 
that used clinical data as input for the classifiers as per [46, 47], we also found cases in which clinical data was used together with other types of data

e.g. 40, 42, 43, $45,48,51,55,56,72$. Fathima and Hundewale [41] and Sanjudev et al. 44 neither provided details about the data set nor the attributes used in their studies.

Thitiprayoonwongse et al. 49 only described the final selected attributes in the data set.

Age, gender, weight and residence (state) were the demographic information present in the data set described in $43,45,48,50,51,55,72]$. The most common clinical data used to classify arboviral diseases were: abdominal pain, fever, temperature, and bleeding.

Table 4 presents the summary of all non-clinical (laboratory and others) data found in this Systematic Literature Review (SLR). As expected, none of the 15 primary studies used only non-clinical data (since these works were excluded from our Systematic Literature Review (SLR)).

The non-clinical data present in the Table 4 is quite diverse. The most common attribute used as input for models was the PLT used in nine studies

- 40,42, $43,45,49,51,55,56$. For model training, most non-clinical data hematological in nature e.g. PLT, WBC and HTC. Dengue IgM, Dengue IgG and Dengue NS1 antigen were used by Gamhbir et al. [43]; ZIKV RT-PCR, TORCHS serology (others except Zika) and neuroimaging reports (US, CT, MRI) were used by Veiga et al. [48]. The biochemical data used in the models were ALT, creatinine and liver size.

Lee et al. 51 compared two cases in relation to the attributes present in their data set: (1) a resource-limited case in which only data available at the time of hospital presentation was used (clinical data), and (2) a well-resourced case in which clinical and laboratory data were used for classification. As per Sub-section 3.3.4 the majority of the best results of the classification of DF, DHF or Chikungunya was obtained using a set of clinical and laboratory data. These results demonstrate that the restricted usage of clinical data for multi-classification may not be as satisfactory as when clinical and non-clinical data is combined. Based on their results, we also highlight that the use of few attributes (they considered only five attributes) is feasible for the classification of DF, DHF and Chikungunya with good performance. Regarding the number of attributes, a similar conclusion was found by Ho et al. 45. They stated that the addition of more attributes did not provide any expressive improvement in the results in any of their models, so the subset with only four attributes was able to provide as much essential information as possible and can be easily collected with minimal cost. Ho et al. 45 highlight two major findings: (1) their "high-sensitivity models can be an effective surveillance tool in the pre-epidemic period" to complement clinical diagnosis, and (2) high-specificity models, as in their proposal, can be exploited to identify laboratory-confirmed dengue cases at outbreak sites for real-time monitoring of epidemic trends.

It is interesting to note that the data sets used are quite different with regard to the number of samples and attributes. In addition, the included studies did not use similar attributes for training and testing their proposed models. In general, the included studies did not describe clinical and non-clinical data in a standardised way, making it difficult to summarise these data without a health professional. Another challenge when analysing the data sets is related to the lack of detailed description in the included studies. Data description and experiment methodology are fundamental for replicability of studies; in half of the cases, there is no information about the model configuration. Additionally, although all studies used data sets, none of these are available for usage further adversely impacting reproducibility. 
Table 3. Summary of all demographic, epidemiological and clinical data presented in data set used by the primary studies.

\begin{tabular}{|c|c|c|c|c|c|c|c|c|c|c|c|c|c|c|c|}
\hline Attributes & 40 & 41] & 42 & $43]$ & 44 & 45 & 55 & 56 & $72]$ & [49] & $46]$ & 50 & [47] & 48] & 51] \\
\hline \multicolumn{16}{|l|}{ Demographic data } \\
\hline Age & $\varnothing$ & - & $\varnothing$ & $\checkmark \star$ & - & $\checkmark \star$ & $\checkmark \star$ & $\checkmark$ & $\varnothing$ & $\varnothing$ & $\varnothing$ & $\checkmark \star$ & $\varnothing$ & $\checkmark$ & $\checkmark$ \\
\hline Gender & $\varnothing$ & - & $\varnothing$ & $\checkmark \star$ & - & $\checkmark$ & $\checkmark \star$ & $\checkmark$ & $\checkmark$ & $\varnothing$ & $\varnothing$ & $\checkmark$ & $\varnothing$ & $\checkmark \star$ & $\checkmark$ \\
\hline Weight & $\varnothing$ & - & $\varnothing$ & $\varnothing$ & - & $\varnothing$ & $\checkmark$ & $\varnothing$ & $\checkmark \star$ & $\varnothing$ & $\varnothing$ & $\varnothing$ & $\varnothing$ & $\varnothing$ & $\varnothing$ \\
\hline Residence (state) & $\varnothing$ & - & $\varnothing$ & $\varnothing$ & - & $\varnothing$ & $\varnothing$ & $\varnothing$ & $\varnothing$ & $\varnothing$ & $\varnothing$ & $\varnothing$ & $\varnothing$ & $\checkmark$ & $\varnothing$ \\
\hline \multicolumn{16}{|l|}{ Epidemiological data } \\
\hline Period of symptoms & $\varnothing$ & - & $\varnothing$ & $\varnothing$ & - & $\varnothing$ & $\varnothing$ & $\checkmark$ & $\varnothing$ & $\varnothing$ & $\varnothing$ & $\checkmark \star$ & $\varnothing$ & $\varnothing$ & $\checkmark \star$ \\
\hline Epidemiological week & $\varnothing$ & - & $\varnothing$ & $\varnothing$ & - & $\checkmark$ & $\varnothing$ & $\varnothing$ & $\varnothing$ & $\varnothing$ & $\varnothing$ & $\varnothing$ & $\varnothing$ & $\checkmark$ & $\varnothing$ \\
\hline Severity & $\varnothing$ & - & $\varnothing$ & $\varnothing$ & - & $\checkmark$ & $\varnothing$ & $\checkmark$ & $\varnothing$ & $\varnothing$ & $\varnothing$ & $\varnothing$ & $\varnothing$ & $\checkmark$ & $\varnothing$ \\
\hline Japanese encephalitis vaccine & $\varnothing$ & - & $\varnothing$ & $\varnothing$ & - & $\varnothing$ & $\varnothing$ & $\varnothing$ & $\varnothing$ & $\checkmark \star$ & $\varnothing$ & $\checkmark$ & $\varnothing$ & $\varnothing$ & $\varnothing$ \\
\hline \multicolumn{16}{|l|}{$\begin{array}{l}\text { Clinical data } \\
\end{array}$} \\
\hline Abdominal pain & $\varnothing$ & - & $\checkmark \star$ & $\checkmark \star$ & - & $\varnothing$ & $\checkmark$ & $\checkmark$ & $\checkmark \star$ & $\checkmark \star$ & $\varnothing$ & $\varnothing$ & $\varnothing$ & $\varnothing$ & $\checkmark$ \\
\hline Fever & $\varnothing$ & - & $\varnothing$ & $\checkmark \star$ & - & $\checkmark \star$ & $\varnothing$ & $\varnothing$ & $\checkmark$ & $\varnothing$ & $\varnothing$ & $\varnothing$ & $\checkmark \star$ & $\varnothing$ & $\checkmark \star$ \\
\hline Headache & $\checkmark$ & - & $\checkmark \star$ & $\checkmark \star$ & - & $\varnothing$ & $\checkmark$ & $\checkmark$ & $\checkmark$ & $\varnothing$ & $\varnothing$ & $\varnothing$ & $\checkmark \star$ & $\varnothing$ & $\checkmark$ \\
\hline Myalgia & $\checkmark$ & - & $\checkmark \star$ & $\checkmark \star$ & - & $\varnothing$ & $\varnothing$ & $\varnothing$ & $\checkmark$ & $\varnothing$ & $\varnothing$ & $\varnothing$ & $\checkmark \star$ & $\varnothing$ & $\checkmark$ \\
\hline Vomiting & $\checkmark$ & - & $\checkmark \star$ & $\checkmark \star$ & - & $\varnothing$ & $\checkmark$ & $\checkmark$ & $\checkmark$ & $\varnothing$ & $\varnothing$ & $\varnothing$ & $\varnothing$ & $\varnothing$ & $\checkmark$ \\
\hline Arthalgya & $\checkmark$ & - & $\checkmark \star$ & $\varnothing$ & - & $\varnothing$ & $\varnothing$ & $\varnothing$ & $\checkmark$ & $\varnothing$ & $\varnothing$ & $\varnothing$ & $\checkmark \star$ & $\varnothing$ & $\varnothing$ \\
\hline Fever (duration) & $\varnothing$ & - & $\varnothing$ & $\varnothing$ & - & $\varnothing$ & $\checkmark$ & $\checkmark$ & $\varnothing$ & $\checkmark \star$ & $\varnothing$ & $\varnothing$ & $\varnothing$ & $\varnothing$ & $\checkmark \star$ \\
\hline Diarrhoea & $\checkmark$ & - & $\checkmark \star$ & $\varnothing$ & - & $\varnothing$ & $\varnothing$ & $\varnothing$ & $\varnothing$ & $\checkmark$ & $\varnothing$ & $\varnothing$ & $\varnothing$ & $\varnothing$ & $\checkmark$ \\
\hline Retroorbital pain & $\checkmark$ & - & $\checkmark \star$ & $\varnothing$ & - & $\varnothing$ & $\varnothing$ & $\checkmark$ & $\varnothing$ & $\varnothing$ & $\varnothing$ & $\varnothing$ & $\varnothing$ & $\varnothing$ & $\varnothing$ \\
\hline Weakness & $\checkmark$ & - & $\varnothing$ & $\checkmark \star$ & - & $\varnothing$ & $\varnothing$ & $\varnothing$ & $\checkmark$ & $\varnothing$ & $\varnothing$ & $\varnothing$ & $\varnothing$ & $\varnothing$ & $\varnothing$ \\
\hline Chills & $\varnothing$ & - & $\varnothing$ & $\checkmark \star$ & - & $\varnothing$ & $\varnothing$ & $\varnothing$ & $\checkmark$ & $\varnothing$ & $\varnothing$ & $\varnothing$ & $\varnothing$ & $\varnothing$ & $\varnothing$ \\
\hline Taste alteration & $\checkmark$ & - & $\checkmark \star$ & $\varnothing$ & - & $\varnothing$ & $\varnothing$ & $\varnothing$ & $\varnothing$ & $\varnothing$ & $\varnothing$ & $\varnothing$ & $\varnothing$ & $\varnothing$ & $\varnothing$ \\
\hline Joint swelling & $\varnothing$ & - & $\varnothing$ & $\varnothing$ & - & $\varnothing$ & $\varnothing$ & $\varnothing$ & $\varnothing$ & $\varnothing$ & $\varnothing$ & $\varnothing$ & $\checkmark \star$ & $\varnothing$ & $\varnothing$ \\
\hline Anorexia & $\checkmark$ & - & $\varnothing$ & $\varnothing$ & - & $\varnothing$ & $\checkmark$ & $\varnothing$ & $\checkmark$ & $\varnothing$ & $\varnothing$ & $\varnothing$ & $\ddot{\varnothing}$ & $\varnothing$ & $\checkmark$ \\
\hline Nausea & $\checkmark$ & - & $\varnothing$ & $\varnothing$ & - & $\varnothing$ & $\checkmark$ & $\varnothing$ & $\checkmark$ & $\varnothing$ & $\varnothing$ & $\varnothing$ & $\varnothing$ & $\varnothing$ & $\checkmark$ \\
\hline Conjunctivitis & $\checkmark$ & - & $\varnothing$ & $\varnothing$ & - & $\varnothing$ & $\varnothing$ & $\varnothing$ & $\checkmark$ & $\varnothing$ & $\varnothing$ & $\varnothing$ & $\varnothing$ & $\varnothing$ & $\varnothing$ \\
\hline Cough & $\varnothing$ & - & $\varnothing$ & $\varnothing$ & - & $\varnothing$ & $\varnothing$ & $\varnothing$ & $\varnothing$ & $\varnothing$ & $\varnothing$ & $\varnothing$ & $\varnothing$ & $\varnothing$ & $\checkmark$ \\
\hline Dizziness & $\varnothing$ & - & $\varnothing$ & $\varnothing$ & - & $\varnothing$ & $\varnothing$ & $\varnothing$ & $\checkmark$ & $\varnothing$ & $\varnothing$ & $\varnothing$ & $\varnothing$ & $\varnothing$ & $\varnothing$ \\
\hline Itching & $\varnothing$ & - & $\varnothing$ & $\varnothing$ & - & $\varnothing$ & $\varnothing$ & $\varnothing$ & $\varnothing$ & $\checkmark$ & $\varnothing$ & $\varnothing$ & $\varnothing$ & $\varnothing$ & $\varnothing$ \\
\hline Jaundice & $\varnothing$ & - & $\varnothing$ & $\varnothing$ & - & $\varnothing$ & $\varnothing$ & $\varnothing$ & $\varnothing$ & $\checkmark$ & $\varnothing$ & $\varnothing$ & $\varnothing$ & $\varnothing$ & $\varnothing$ \\
\hline Sore throat & $\varnothing$ & - & $\varnothing$ & $\varnothing$ & - & $\varnothing$ & $\varnothing$ & $\varnothing$ & $\varnothing$ & $\varnothing$ & $\varnothing$ & $\varnothing$ & $\varnothing$ & $\varnothing$ & $\checkmark$ \\
\hline Skin sensitivity & $\checkmark-$ & - & $\varnothing$ & $\varnothing$ & - & $\varnothing$ & $\varnothing$ & $\varnothing$ & $\varnothing$ & $\varnothing$ & $\varnothing$ & $\varnothing$ & $\varnothing$ & $\varnothing$ & $\varnothing$ \\
\hline \multicolumn{16}{|l|}{${ }^{-}-\overline{\text { Signs }}$} \\
\hline Temperature & $\checkmark \star$ & - & $\checkmark \star$ & $\checkmark \star$ & - & $\checkmark$ & $\varnothing$ & $\checkmark$ & $\varnothing$ & $\checkmark$ & $\checkmark \star$ & $\varnothing$ & $\varnothing$ & $\varnothing$ & $\checkmark$ \\
\hline Bleeding & $\checkmark$ & - & $\varnothing$ & $\varnothing$ & - & $\varnothing$ & $\checkmark$ & $\checkmark$ & $\checkmark \star$ & $\checkmark \star$ & $\checkmark \star$ & $\varnothing$ & $\varnothing$ & $\varnothing$ & $\checkmark \star$ \\
\hline Tornikuet test & $\varnothing$ & - & $\varnothing$ & $\varnothing$ & - & $\varnothing$ & $\checkmark \star$ & $\varnothing$ & $\varnothing$ & $\checkmark$ & $\checkmark \star$ & $\checkmark \star$ & $\varnothing$ & $\varnothing$ & $\varnothing$ \\
\hline Hepatomegaly & $\varnothing$ & - & $\varnothing$ & $\varnothing$ & - & $\varnothing$ & $\hat{\varnothing}$ & $\checkmark$ & $\checkmark \star$ & $\checkmark \star$ & $\varnothing$ & $\checkmark$ & $\varnothing$ & $\varnothing$ & $\varnothing$ \\
\hline Shock & $\varnothing$ & - & $\varnothing$ & $\varnothing$ & - & $\varnothing$ & $\varnothing$ & $\varnothing$ & $\varnothing$ & $\checkmark \star$ & $\varnothing$ & $\checkmark \star$ & $\varnothing$ & $\varnothing$ & $\varnothing$ \\
\hline Heart rate & $\checkmark$ & - & $\varnothing$ & $\checkmark \star$ & - & $\checkmark$ & $\varnothing$ & $\checkmark$ & $\varnothing$ & $\hat{\checkmark}$ & $\varnothing$ & $\varnothing$ & $\varnothing$ & $\varnothing$ & $\checkmark$ \\
\hline Rash & $\checkmark$ & - & $\varnothing$ & $\varnothing$ & - & $\varnothing$ & $\varnothing$ & $\checkmark$ & $\checkmark$ & $\checkmark \star$ & $\varnothing$ & $\varnothing$ & $\varnothing$ & $\checkmark t$ & $\checkmark$ \\
\hline Pleural effusion & $\varnothing$ & - & $\varnothing$ & $\varnothing$ & - & $\varnothing$ & $\varnothing$ & $\varnothing$ & $\varnothing$ & $\checkmark$ & $\varnothing$ & $\checkmark \star$ & $\varnothing$ & $\varnothing$ & $\varnothing$ \\
\hline Ascites & $\varnothing$ & - & $\varnothing$ & $\varnothing$ & - & $\varnothing$ & $\varnothing$ & $\varnothing$ & $\varnothing$ & $\checkmark$ & $\varnothing$ & $\checkmark \star$ & $\varnothing$ & $\varnothing$ & $\varnothing$ \\
\hline Glasgow Coma Score & $\varnothing$ & - & $\varnothing$ & $\varnothing$ & - & $\varnothing$ & $\varnothing$ & $\checkmark \star$ & $\varnothing$ & $\varnothing$ & $\varnothing$ & $\hat{\varnothing}$ & $\varnothing$ & $\varnothing$ & $\varnothing$ \\
\hline Gestacional age & $\varnothing$ & - & $\varnothing$ & $\varnothing$ & - & $\varnothing$ & $\varnothing$ & $\varnothing$ & $\varnothing$ & $\varnothing$ & $\varnothing$ & $\varnothing$ & $\varnothing$ & $\checkmark \star$ & $\varnothing$ \\
\hline Head circumference & $\varnothing$ & - & $\varnothing$ & $\varnothing$ & - & $\varnothing$ & $\varnothing$ & $\varnothing$ & $\varnothing$ & $\varnothing$ & $\varnothing$ & $\varnothing$ & $\varnothing$ & $\checkmark \star$ & $\varnothing$ \\
\hline Plasma leakage & $\varnothing$ & - & $\varnothing$ & $\varnothing$ & - & $\varnothing$ & $\varnothing$ & $\varnothing$ & $\varnothing$ & $\checkmark \star$ & $\varnothing$ & $\varnothing$ & $\varnothing$ & $\checkmark \star$ & $\varnothing$ \\
\hline Blood Pressure & $\varnothing$ & - & $\varnothing$ & $\varnothing$ & - & $\checkmark$ & $\varnothing$ & $\varnothing$ & $\varnothing$ & $\checkmark$ & $\varnothing$ & $\varnothing$ & $\varnothing$ & $\varnothing$ & $\varnothing$ \\
\hline Respiratory rate & $\varnothing$ & - & $\varnothing$ & $\varnothing$ & - & $\checkmark$ & $\varnothing$ & $\checkmark$ & $\varnothing$ & $\checkmark$ & $\varnothing$ & $\varnothing$ & $\varnothing$ & $\varnothing$ & $\varnothing$ \\
\hline Flush face & $\varnothing$ & - & $\varnothing$ & $\varnothing$ & - & $\varnothing$ & $\varnothing$ & $\varnothing$ & $\checkmark$ & $\varnothing$ & $\varnothing$ & $\varnothing$ & $\varnothing$ & $\varnothing$ & $\varnothing$ \\
\hline Palpable lymphadenopathy & $\checkmark$ & - & $\varnothing$ & $\varnothing$ & - & $\varnothing$ & $\varnothing$ & $\varnothing$ & $\varnothing$ & $\checkmark$ & $\varnothing$ & $\varnothing$ & $\varnothing$ & $\varnothing$ & $\varnothing$ \\
\hline Birthwieght & $\varnothing$ & - & $\varnothing$ & $\varnothing$ & - & $\varnothing$ & $\varnothing$ & $\varnothing$ & $\varnothing$ & $\varnothing$ & $\varnothing$ & $\varnothing$ & $\varnothing$ & $\checkmark$ & $\varnothing$ \\
\hline Capillary refill time & $\varnothing$ & & $\varnothing$ & $\varnothing$ & & $\varnothing$ & $\varnothing$ & $\checkmark$ & $\varnothing$ & $\varnothing$ & $\varnothing$ & $\varnothing$ & $\varnothing$ & $\varnothing$ & $\varnothing$ \\
\hline Comorbidities & & & & & & & & & & & & & & & \\
\hline Hypertension (HT) & $\varnothing$ & - & $\varnothing$ & $\varnothing$ & - & $\checkmark$ & $\varnothing$ & $\varnothing$ & $\varnothing$ & $\varnothing$ & $\varnothing$ & $\varnothing$ & $\varnothing$ & $\varnothing$ & $\checkmark$ \\
\hline NCDs (except HT) & $\varnothing$ & - & $\varnothing$ & $\varnothing$ & - & $\checkmark$ & $\varnothing$ & $\varnothing$ & $\varnothing$ & $\varnothing$ & $\varnothing$ & $\varnothing$ & $\varnothing$ & $\varnothing$ & $\varnothing$ \\
\hline Upper respiratory infection & $\varnothing$ & - & $\varnothing$ & $\varnothing$ & - & $\varnothing$ & $\varnothing$ & $\varnothing$ & $\varnothing$ & $\checkmark$ & $\varnothing$ & $\varnothing$ & $\varnothing$ & $\varnothing$ & $\varnothing$ \\
\hline
\end{tabular}

$\checkmark$ : data available in the data set; $\varnothing$ : data not available in the data set; $\star$ : data used as input for the models; -: data set not described; $t$ : maternal history of rash. Some atributes were generalized based

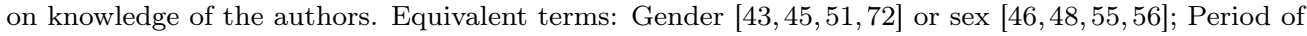
symptoms 50 56 or time since onset 51; Severity (non-hospitalized, hospitalized, ICU admission and death); Myalgia 51.72, bodyache 43.72 or muscle pain 40 42 47]; Artralgya [51] or joint

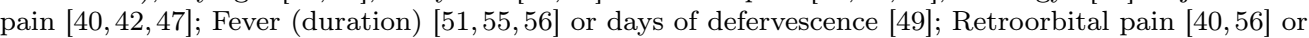
pain behind eyes [42]; Weakness [4] 56] or drowsiness [40]; Taste alteration [40] or metallic taste [42]; Anorexia 51,55.72 or loss of apetite [40; Conjunctivitis 49] or red eyes 40];

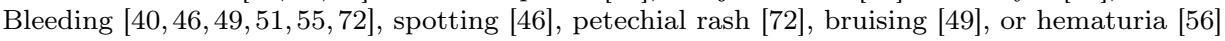
Rumpel-Leed test 50, R/L test 50 or tornikuet test 46 49 55 ; Hepatomegaly 50 72, grown liver 49 or liver enlargement 56]; Heart rate 43, 45], pulse rate 40|56 or tachycardia 51]; Rash 40 49 51 56. macular 72, or maternal history of rash 48; Pleural effusion 49 50 or pleural effusion index 55;

Respiratory rate 45 56] or dyspnea 49]; Palpable lymphadenopathy 40] or lymph node enlargement 49]; Non-communicable diseases (NCDs) include heart disease, stroke, renal injury, severe liver disease or cancer. 
Table 4. Summary of all non-clinical data (laboratory and others) presented in the data set used by the primary studies.

\begin{tabular}{|c|c|c|c|c|c|c|c|c|c|c|c|c|c|c|c|}
\hline Attributes & 40 & 41] & 42 & 43] & 44 & 45] & 55 & 56] & 72 & 49] & 46 & 50] & 47 & 48 & 51 \\
\hline \multicolumn{16}{|l|}{$\begin{array}{l}\text { Laboratory data } \\
-\overline{\text { Hermatologicalo }}\end{array}$} \\
\hline PLT & $\checkmark \star$ & - & $\checkmark \star$ & $\checkmark \star$ & - & $\checkmark \star$ & $\checkmark \star$ & $\checkmark \star$ & $\checkmark$ & $\checkmark \star$ & $\varnothing$ & $\checkmark \star$ & $\varnothing$ & $\varnothing$ & $\checkmark \star$ \\
\hline WBC & $\checkmark \star$ & - & $\checkmark \star$ & $\varnothing$ & - & $\checkmark \star$ & $\checkmark \star$ & $\checkmark$ & $\varnothing$ & $\checkmark \star$ & $\varnothing$ & $\varnothing$ & $\varnothing$ & $\varnothing$ & $\checkmark \star$ \\
\hline HTC & $\checkmark \star$ & - & $\varnothing$ & $\varnothing$ & - & $\varnothing$ & $\checkmark \star$ & $\checkmark \star$ & $\checkmark$ & $\checkmark \star$ & $\varnothing$ & $\checkmark \star$ & $\varnothing$ & $\varnothing$ & $\checkmark$ \\
\hline LYMPH & $\checkmark \star$ & - & $\varnothing$ & $\varnothing$ & - & $\varnothing$ & $\checkmark$ & $\checkmark$ & $\varnothing$ & $\checkmark \star$ & $\varnothing$ & $\varnothing$ & $\varnothing$ & $\varnothing$ & $\checkmark$ \\
\hline HGB & $\checkmark$ & - & $\checkmark \star$ & $\varnothing$ & - & $\checkmark$ & $\varnothing$ & $\varnothing$ & $\varnothing$ & $\varnothing$ & $\varnothing$ & $\checkmark \star$ & $\varnothing$ & $\varnothing$ & $\checkmark$ \\
\hline NEUT & $\checkmark \star$ & - & $\varnothing$ & $\varnothing$ & - & $\varnothing$ & $\checkmark$ & $\checkmark$ & $\varnothing$ & $\varnothing$ & $\varnothing$ & $\varnothing$ & $\varnothing$ & $\varnothing$ & $\checkmark$ \\
\hline LYMPH\% & $\checkmark$ & - & $\varnothing$ & $\varnothing$ & - & $\varnothing$ & $\checkmark \star$ & $\varnothing$ & $\varnothing$ & $\varnothing$ & $\varnothing$ & $\varnothing$ & $\varnothing$ & $\varnothing$ & $\checkmark$ \\
\hline NEUT\% & $\checkmark$ & - & $\varnothing$ & $\varnothing$ & - & $\varnothing$ & $\checkmark \star$ & $\varnothing$ & $\varnothing$ & $\varnothing$ & $\varnothing$ & $\varnothing$ & $\varnothing$ & $\varnothing$ & $\varnothing$ \\
\hline MONO\% & $\checkmark$ & - & $\varnothing$ & $\varnothing$ & - & $\varnothing$ & $\checkmark \star$ & $\varnothing$ & $\varnothing$ & $\varnothing$ & $\varnothing$ & $\varnothing$ & $\varnothing$ & $\varnothing$ & $\varnothing$ \\
\hline ALYMPH\% & $\varnothing$ & - & $\varnothing$ & $\varnothing$ & - & $\varnothing$ & $\checkmark$ & $\varnothing$ & $\varnothing$ & $\checkmark$ & $\varnothing$ & $\varnothing$ & $\varnothing$ & $\varnothing$ & $\checkmark$ \\
\hline MONO & $\checkmark$ & - & $\varnothing$ & $\varnothing$ & - & $\varnothing$ & $\checkmark$ & $\varnothing$ & $\varnothing$ & $\varnothing$ & $\varnothing$ & $\varnothing$ & $\varnothing$ & $\varnothing$ & $\checkmark$ \\
\hline EOSBAS & $\checkmark$ & - & $\varnothing$ & $\varnothing$ & - & $\varnothing$ & $\varnothing$ & $\varnothing$ & $\varnothing$ & $\varnothing$ & $\varnothing$ & $\varnothing$ & $\varnothing$ & $\varnothing$ & $\varnothing$ \\
\hline EOSBAS\% & $\checkmark$ & - & $\varnothing$ & $\varnothing$ & - & $\varnothing$ & $\varnothing$ & $\varnothing$ & $\varnothing$ & $\varnothing$ & $\varnothing$ & $\varnothing$ & $\varnothing$ & $\varnothing$ & $\varnothing$ \\
\hline $\mathrm{MCH}$ & $\checkmark$ & - & $\varnothing$ & $\varnothing$ & - & $\varnothing$ & $\varnothing$ & $\varnothing$ & $\varnothing$ & $\varnothing$ & $\varnothing$ & $\varnothing$ & $\varnothing$ & $\varnothing$ & $\varnothing$ \\
\hline $\mathrm{MCHC}$ & $\checkmark$ & - & $\varnothing$ & $\varnothing$ & - & $\varnothing$ & $\varnothing$ & $\varnothing$ & $\varnothing$ & $\varnothing$ & $\varnothing$ & $\varnothing$ & $\varnothing$ & $\varnothing$ & $\varnothing$ \\
\hline $\mathrm{MCV}$ & $\checkmark$ & - & $\varnothing$ & $\varnothing$ & - & $\varnothing$ & $\varnothing$ & $\varnothing$ & $\varnothing$ & $\varnothing$ & $\varnothing$ & $\varnothing$ & $\varnothing$ & $\varnothing$ & $\varnothing$ \\
\hline MPV & $\checkmark$ & - & $\varnothing$ & $\varnothing$ & - & $\varnothing$ & $\varnothing$ & $\varnothing$ & $\varnothing$ & $\varnothing$ & $\varnothing$ & $\varnothing$ & $\varnothing$ & $\varnothing$ & $\varnothing$ \\
\hline PDW & $\checkmark$ & - & $\varnothing$ & $\varnothing$ & - & $\varnothing$ & $\varnothing$ & $\varnothing$ & $\varnothing$ & $\varnothing$ & $\varnothing$ & $\varnothing$ & $\varnothing$ & $\varnothing$ & $\varnothing$ \\
\hline PLCR & $\checkmark$ & - & $\varnothing$ & $\varnothing$ & - & $\varnothing$ & $\varnothing$ & $\varnothing$ & $\varnothing$ & $\varnothing$ & $\varnothing$ & $\varnothing$ & $\varnothing$ & $\varnothing$ & $\varnothing$ \\
\hline $\mathrm{RBC}$ & $\checkmark$ & - & $\varnothing$ & $\varnothing$ & - & $\varnothing$ & $\varnothing$ & $\varnothing$ & $\varnothing$ & $\varnothing$ & $\varnothing$ & $\varnothing$ & $\varnothing$ & $\varnothing$ & $\varnothing$ \\
\hline RDW & $\checkmark$ & - & $\varnothing$ & $\varnothing$ & - & $\varnothing$ & $\varnothing$ & $\varnothing$ & $\varnothing$ & $\varnothing$ & $\varnothing$ & $\varnothing$ & $\varnothing$ & $\varnothing$ & $\varnothing$ \\
\hline \multicolumn{16}{|l|}{ Biochémical } \\
\hline ALT & $\varnothing$ & - & $\varnothing$ & $\varnothing$ & - & $\varnothing$ & $\checkmark \star$ & $\checkmark$ & $\checkmark$ & $\checkmark \star$ & $\varnothing$ & $\varnothing$ & $\varnothing$ & $\varnothing$ & $\checkmark$ \\
\hline AST & $\varnothing$ & - & $\varnothing$ & $\varnothing$ & - & $\varnothing$ & $\checkmark \star$ & $\varnothing$ & $\checkmark$ & $\checkmark \star$ & $\varnothing$ & $\varnothing$ & $\varnothing$ & $\varnothing$ & $\checkmark$ \\
\hline Creatinine & $\varnothing$ & - & $\varnothing$ & $\varnothing$ & - & $\varnothing$ & $\varnothing$ & $\checkmark \star$ & $\varnothing$ & $\varnothing$ & $\varnothing$ & $\varnothing$ & $\varnothing$ & $\varnothing$ & $\checkmark$ \\
\hline Albumin & $\varnothing$ & - & $\varnothing$ & $\varnothing$ & - & $\varnothing$ & $\checkmark$ & $\varnothing$ & $\varnothing$ & $\checkmark$ & $\varnothing$ & $\varnothing$ & $\varnothing$ & $\varnothing$ & $\checkmark$ \\
\hline Protein & $\varnothing$ & - & $\varnothing$ & $\varnothing$ & - & $\varnothing$ & $\varnothing$ & $\varnothing$ & $\varnothing$ & $\checkmark$ & $\varnothing$ & $\checkmark$ & $\varnothing$ & $\varnothing$ & $\checkmark$ \\
\hline Urea & $\varnothing$ & - & $\varnothing$ & $\varnothing$ & - & $\varnothing$ & $\varnothing$ & $\checkmark$ & $\varnothing$ & $\varnothing$ & $\varnothing$ & $\varnothing$ & $\varnothing$ & $\varnothing$ & $\checkmark$ \\
\hline ALP & $\varnothing$ & - & $\varnothing$ & $\varnothing$ & - & $\varnothing$ & $\varnothing$ & $\varnothing$ & $\varnothing$ & $\varnothing$ & $\varnothing$ & $\varnothing$ & $\varnothing$ & $\varnothing$ & $\checkmark$ \\
\hline Bilirubun & $\varnothing$ & - & $\varnothing$ & $\varnothing$ & - & $\varnothing$ & $\varnothing$ & $\varnothing$ & $\varnothing$ & $\varnothing$ & $\varnothing$ & $\varnothing$ & $\varnothing$ & $\varnothing$ & $\checkmark$ \\
\hline Potassium & $\varnothing$ & - & $\varnothing$ & $\varnothing$ & - & $\varnothing$ & $\varnothing$ & $\varnothing$ & $\varnothing$ & $\varnothing$ & $\varnothing$ & $\varnothing$ & $\varnothing$ & $\varnothing$ & $\checkmark$ \\
\hline \multirow{2}{*}{\multicolumn{16}{|c|}{ 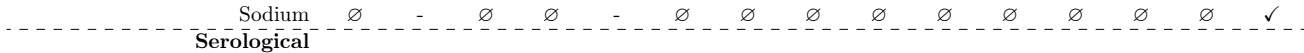 }} \\
\hline & & & & & & & & & & & & & & & \\
\hline Dengue IgM (ELISA) & $\varnothing$ & - & $\varnothing$ & $\checkmark \star$ & - & $\checkmark$ & $\checkmark$ & $\checkmark^{*}$ & $\varnothing$ & $\varnothing$ & $\varnothing$ & $\checkmark$ & $\varnothing$ & $\varnothing$ & $\varnothing$ \\
\hline Dengue IgG (ELISA) & $\checkmark$ & - & $\varnothing$ & $\checkmark \star$ & - & $\varnothing$ & $\checkmark$ & $\varnothing$ & $\varnothing$ & $\varnothing$ & $\varnothing$ & $\checkmark$ & $\varnothing$ & $\varnothing$ & $\varnothing$ \\
\hline Dengue NS1 antigen (ELISA) & $\varnothing$ & - & $\varnothing$ & $\checkmark \star$ & - & $\checkmark$ & $\varnothing$ & $\checkmark$ & $\varnothing$ & $\varnothing$ & $\varnothing$ & $\varnothing$ & $\varnothing$ & $\varnothing$ & $\varnothing$ \\
\hline TORCHS serology $\ddagger$ & $\varnothing$ & - & $\varnothing$ & $\varnothing$ & - & $\varnothing$ & $\varnothing$ & $\varnothing$ & $\varnothing$ & $\varnothing$ & $\varnothing$ & $\varnothing$ & $\varnothing$ & $\checkmark \star$ & $\varnothing$ \\
\hline Zika serology & $\varnothing$ & - & $\varnothing$ & $\varnothing$ & - & $\varnothing$ & $\varnothing$ & $\varnothing$ & $\varnothing$ & $\varnothing$ & $\varnothing$ & $\varnothing$ & $\varnothing$ & $\checkmark \star$ & $\varnothing$ \\
\hline Dengue antiboties (HAI) & $\varnothing$ & - & $\varnothing$ & $\varnothing$ & - & $\varnothing$ & $\checkmark$ & $\varnothing$ & $\varnothing$ & $\varnothing$ & $\varnothing$ & $\varnothing$ & $\varnothing$ & $\varnothing$ & $\varnothing$ \\
\hline \multirow{2}{*}{\multicolumn{16}{|c|}{$\begin{array}{l}\text { JEV and Dengue IgM (ELISA) } \\
-{ }_{-}-{ }_{-} \text {Molecular biology }\end{array}$}} \\
\hline & & & & & & & & & & & & & & & \\
\hline Dengue RT/PCR & $\checkmark$ & - & $\varnothing$ & $\varnothing$ & - & $\checkmark$ & $\varnothing$ & $\varnothing$ & $\varnothing$ & $\varnothing$ & $\varnothing$ & $\varnothing$ & $\varnothing$ & $\varnothing$ & $\checkmark$ \\
\hline Dengue viral load & $\checkmark$ & - & $\varnothing$ & $\varnothing$ & - & $\checkmark$ & $\varnothing$ & $\varnothing$ & $\varnothing$ & $\varnothing$ & $\varnothing$ & $\varnothing$ & $\varnothing$ & $\varnothing$ & $\varnothing$ \\
\hline \multirow{2}{*}{\multicolumn{16}{|c|}{ 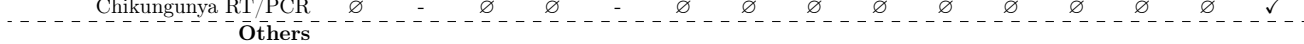 }} \\
\hline & & & & & & & & & & & & & & & \\
\hline Dengue antigen NS1 & $\varnothing$ & - & $\varnothing$ & $\checkmark \star$ & - & $\checkmark$ & $\varnothing$ & $\varnothing$ & $\varnothing$ & $\varnothing$ & $\varnothing$ & $\varnothing$ & $\varnothing$ & $\varnothing$ & $\varnothing$ \\
\hline Urine protein & $\varnothing$ & - & $\varnothing$ & $\varnothing$ & - & $\varnothing$ & $\varnothing$ & $\checkmark \star$ & $\varnothing$ & $\varnothing$ & $\varnothing$ & $\varnothing$ & $\varnothing$ & $\varnothing$ & $\varnothing$ \\
\hline Blood in stool & $\varnothing$ & - & $\varnothing$ & $\varnothing$ & - & $\varnothing$ & $\varnothing$ & $\checkmark$ & $\varnothing$ & $\varnothing$ & $\varnothing$ & $\varnothing$ & $\varnothing$ & $\varnothing$ & $\varnothing$ \\
\hline Dengue viral isolation & $\varnothing$ & - & $\varnothing$ & $\varnothing$ & - & $\varnothing$ & $\checkmark$ & $\varnothing$ & $\varnothing$ & $\varnothing$ & $\varnothing$ & $\varnothing$ & $\varnothing$ & $\varnothing$ & $\varnothing$ \\
\hline Urine red blood cells & $\varnothing$ & - & $\varnothing$ & $\varnothing$ & - & $\varnothing$ & $\varnothing$ & $\checkmark$ & $\varnothing$ & $\varnothing$ & $\varnothing$ & $\varnothing$ & $\varnothing$ & $\varnothing$ & $\varnothing$ \\
\hline \multicolumn{16}{|l|}{ Medical imaging } \\
\hline Neuroimaging report & $\varnothing$ & - & $\varnothing$ & $\varnothing$ & - & $\varnothing$ & $\varnothing$ & $\varnothing$ & $\varnothing$ & $\varnothing$ & $\varnothing$ & $\varnothing$ & $\varnothing$ & $\checkmark \star$ & $\varnothing$ \\
\hline Chest radiography & $\varnothing$ & - & $\varnothing$ & $\varnothing$ & - & $\varnothing$ & $\checkmark$ & $\varnothing$ & $\varnothing$ & $\varnothing$ & $\varnothing$ & $\varnothing$ & $\varnothing$ & $\varnothing$ & $\varnothing$ \\
\hline \multicolumn{16}{|l|}{ Bioeletrical impedance } \\
\hline Extracellular Water & $\varnothing$ & - & $\varnothing$ & $\varnothing$ & - & $\varnothing$ & $\varnothing$ & $\varnothing$ & $\checkmark \star$ & $\varnothing$ & $\varnothing$ & $\varnothing$ & $\varnothing$ & $\varnothing$ & $\varnothing$ \\
\hline Body Cell Mass & $\varnothing$ & - & $\varnothing$ & $\varnothing$ & - & $\varnothing$ & $\varnothing$ & $\varnothing$ & $\checkmark \star$ & $\varnothing$ & $\varnothing$ & $\varnothing$ & $\varnothing$ & $\varnothing$ & $\varnothing$ \\
\hline Reactance & $\varnothing$ & - & $\varnothing$ & $\varnothing$ & - & $\varnothing$ & $\varnothing$ & $\varnothing$ & $\checkmark \star$ & $\varnothing$ & $\varnothing$ & $\varnothing$ & $\varnothing$ & $\varnothing$ & $\varnothing$ \\
\hline Others $\ddagger$ & $\varnothing$ & - & $\varnothing$ & $\varnothing$ & - & $\varnothing$ & $\varnothing$ & $\varnothing$ & $\checkmark$ & $\varnothing$ & $\varnothing$ & $\varnothing$ & $\varnothing$ & $\varnothing$ & $\varnothing$ \\
\hline \multicolumn{16}{|c|}{ 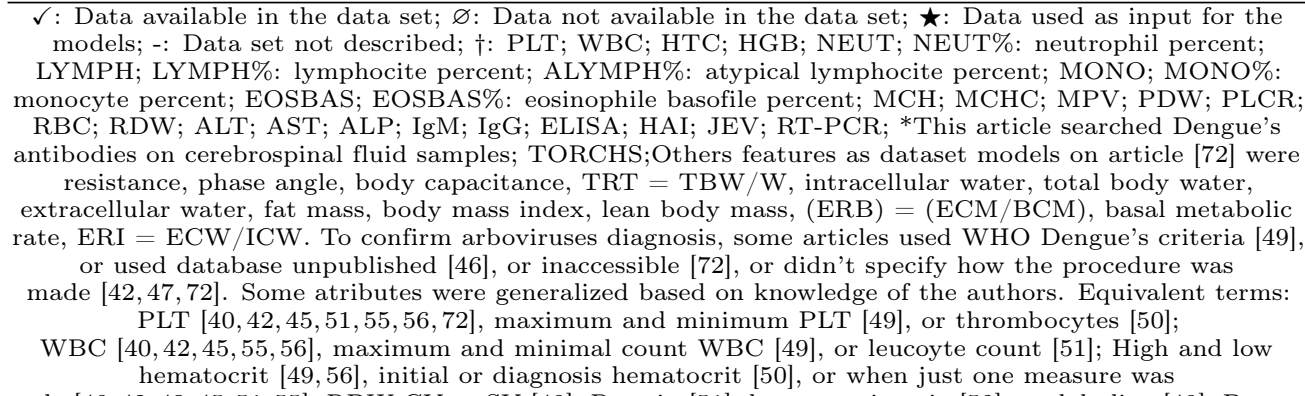 } \\
\hline
\end{tabular}

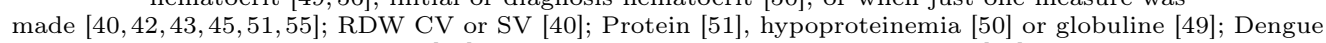
viral load 45] or Crossover threshold of DENV RT-CR 40]. 


\subsection{What are the metrics being used to evaluate the performance of the Machine Learning and Deep Learning techniques?}

The common metrics used to evaluate a classifier are calculated based on a confusion matrix. The confusion matrix is a cross table that records the number of occurrences between the true classification and the classification predicted by the model [82]. It is composed of four values:

- True Positive (TP): The number of values of the principal class that the model predicts right.

- False Positive (FP): The number of values of the principal class that the model predicts wrong.

- True Negative (TN): The number of values of the secondary class that the model predicts right.

- False Negative (FN): The number of values of the secondary class that the model predicts wrong.

Figure 4 presents the metrics used to evaluate the proposed models in the literature. Some works used more than one metric, so some of them are duplicated in the graph. The evaluation metrics used by the works found in this Systematic Literature Review (SLR) are: sensitivity, accuracy, specificity, precision, Receiver Operating Characteristic (ROC) and Area Under the Curve (AUC) and F1-score. Sensitivity and accuracy were used in most works. Next, we describe each metrics.

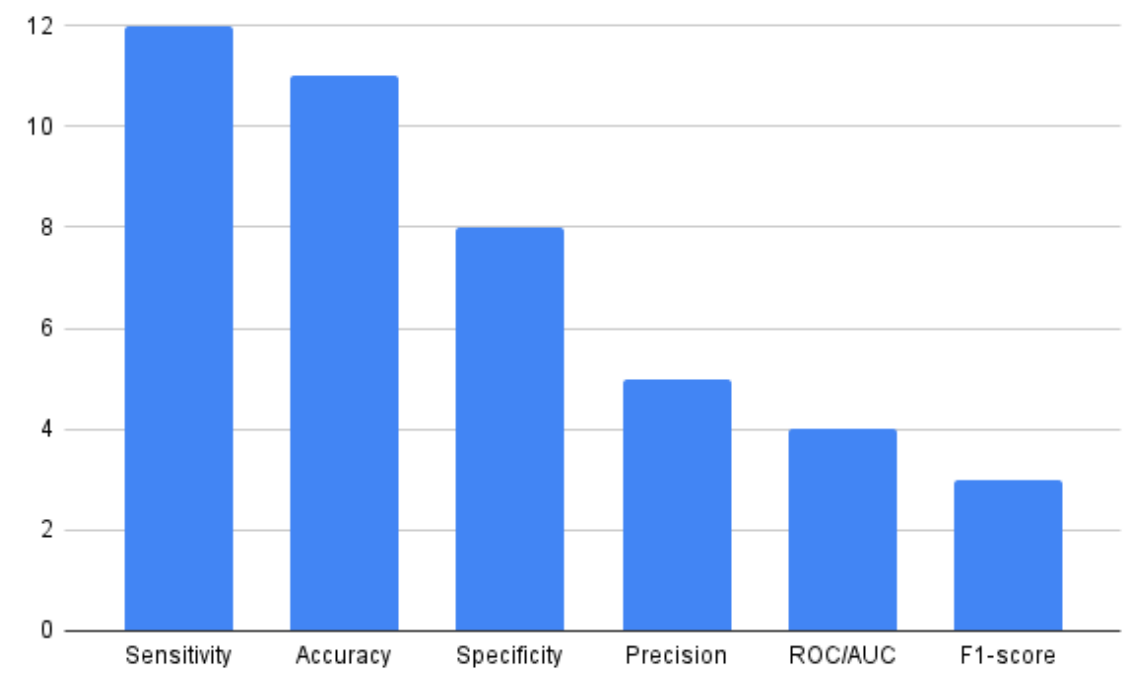

Figure 4. Metrics used to evaluate the models proposed in the literature.

\subsubsection{Sensitivity}

Sensitivity, also know as recall, was used by seven works: [45], [40], [49], 441], [51], [56], [42], [43], [46], 44], [50] and [48]. It defines how well a model correctly predicted TP cases, being calculated as the number of TP divided by the sum of TP and FN, as shown in Eq. 1. 


$$
\text { sensitivity }=\frac{T P}{T P+F N}
$$

\subsubsection{Accuracy}

Accuracy was the most common metric used among the works found in this Systematic Literature Review (SLR): 45], 40, 72], [49], [42, [41], [43], [46], [44], [50] and [48]. It is used to find out how much a model is right. It is calculated as the sum of TP and TN divided by the total of samples, as shown in Eq. 2

$$
\text { accuracy }=\frac{T P+T N}{T P+T N+F P+F N}
$$

\subsubsection{Specificity}

Specificity was used by five works found in this Systematic Literature Review (SLR): 45], [40], [49], [41], [51], [56], [43] and [44]. This metric determines how well the model correctly predicted TN cases. It is calculated by the number of TN divided by the sum of TN and FP. Being calculated as presented in Eq. 3.

$$
\text { specificity }=\frac{T N}{T N+F P}
$$

\subsubsection{Precision}

Precision was used in [56], [42, [46], 50] and [48]. This metric defines how many cases classified as TP actually are TP, and is calculated as the number of TP divided by the sum of TP and FP, as shown in Eq. 4

$$
\text { precision }=\frac{T P}{T P+F P}
$$

\subsubsection{ROC and AUC}

These metrics were used in four works, [51], [46], [44] and [47]. The ROC curve is a graph to analyse the discriminating ability of the model, that is, how well the model is able to divide between two classes. It is a graph with the True Positive Rate (TPR), the sensitivity, in the $x$ axis and the False Positive Rate (FPR), the complement of the specificity, in the $y$ axis. Based on ROC, it is possible to calculate the Area Under the Curve (AUC). The AUC summarises the ROC curve in a single value, aggregating all the ROC thresholds. Its result varies between 0 and 1; an AUC of 0.5 represents a test without discriminating ability, while an AUC of 1.0 represents a test with perfect discrimination 83 .

\subsubsection{F1-Score}

The F1-score is the harmonic mean between two metrics: precision and sensitivity. It is used when the objective is to seek a balance between these two metrics, being calculated as presented in Eq. 5. This metric was used in [42], [46] and 48].

$$
F 1-\text { score }=2 \times \frac{\text { precision } \times \text { sensitivity }}{\text { precision }+ \text { sensitivity }}
$$

To address the imbalanced data issues mentioned earlier, more informative assessment metrics can be used to evaluate models. These include AUC, precision-recall curves and cost curves. Notwithstanding suspected imbalances, most of the included 
studies employed traditional metrics such as accuracy, sensitivity and specificity. Relatively few (3) used AUC 40,44,47.

\section{Discussion}

In this SLR on the use of ML and DL to support the clinical diagnosis of arboviral diseases, we found 963 publications, 15 of which fulfilled the inclusion criteria and were subsequently analysed in detail. We have reported our findings in five main categories: (1) disease focus, (2) machine learning and deep learning technique, (3) machine learning and deep learning model design, (4) data sets and attributes, and (5) evaluation metrics. Comparing the selected studies, even within these categories, due to the variation in focal disease and region, ML and DL technique, and ML and DL model configuration. Results are not uniformly presented. Six of the

studies [41,42, 44, 46, 51, 56 did not provide sufficient detail on their proposed models, none of the selected studies provided access to their data, and only one study [48] provided details of their models online for download.

Firstly, given the low number of studies, it is important to note that there is a clearly a dearth of research in the use of ML and DL to support clinical diagnosis of arboviral diseases as a whole. This paucity of research is further exacerbated when one considers that only three arboviral diseases (Dengue, Chikungunya, and Zika) feature in the selected studies, and most of the papers (12) focus on one disease, Dengue.

Arboviruses, such as yellow fever, which did not feature in any of the selected studies have significant burdens. 47 countries in Africa, Central and South America have regions that are endemic for yellow fever. For example, in 2013, the burden of yellow fever in Africa alone was estimated at 84,000-170,000 severe cases and 29,000-60,000 deaths. While Dengue, Chikungunya, and Zika undoubtedly require further study, there exists a significant need for research in the wider spectrum of arboviral diseases. In particular, given the similarity in symptoms across arboviruses, there is noteworthy lack of research on ML and DL to support differential diagnosis using clinical data. While some studies pursued multi-class classification, this was limited. Again, this may prove to be a fruitful avenue of research.

Systems identified for ML and DL diagnosis of arboviruses using clinical data were generally found to be effective. However, these findings must be tempered with caution. In some cases, for example Sanjudevi and Savitha 44] and Sajana et al. [42], the extremely high performance metrics suggest model overfitting. In both cases, there is a lack of detail on model configuration, feature selection, and hyperparameter optimisation. In the case of Sajana et al. [42], the data set is very small.

There is an increasing range of both ML and DL techniques that can be applied effectively in bio-medical use cases. Our findings suggest that, to date, these techniques have not been explored fully with a significant emphasis on tree-based models. In particular, we note that only one paper made use of a deep learning architecture, a CNN [45], and no papers made use of ensemble methods combining DL and ML. DL methods are attracting significant attention in health due to their improved performance, reduced manual feature engineering, and ability to accommodate the large and complex data sets characteristic of the wider health domain, when compared to traditional machine learning [84]. Future research should consider benchmarking the performance of a wider set of DL architectures, both discretely and as part of ensembles, including RNNs, DBNs, and DSNs, amongst others. In particular, LSTM and GRU, both types of RNNs, may prove fruitful.

While ML and DL techniques represent a significant opportunity for research and practice, they pose their own challenges. Two significant challenges are transparency and data availability. ML and DL are often referred to as black box models as their 
inner workings is too complex for a human to comprehend. As such they have been criticised for their lack of interpretability, comprehensibility, and transparency. For legal, ethical, and scientific reasons, this is a significant issue for high stakes decisions such as clinical diagnosis 85 86. As such, there has been numerous calls for research on explainable machine learning and AI (sometimes referred to as XAI) 85 87 and some small but significant progress has been made in the use of XAI in clinical diagnosis, albeit not in arboviruses, the focus of this paper (see for example [88]).

A second significant challenge in the diagnosis of arboviral diseases using clinical data is more logistical and is related to the size of available data sets. Having a sufficient data set to train and validate ML and DL models is critical. Firstly, none of the studies in the sample explicitly describe or discuss imbalanced data, a common feature across the studies. Secondly, our findings suggest that most of the data sets in the selected studies were relatively small. One might argue, too small. The largest, presented in Fahmi et al. 50 comprised only 14,019 records, while the smallest comprised 20 records 42 . This both impacts confidence in results and generalisability but also has practical implications for their future operational use. Overcoming these problems requires a coordinated effort by health surveillance systems and researchers worldwide and greater sharing of clinical data.

Feature selection and hyperparameter optimisation are key steps in the selection and optimisation of ML and DL models. A significant issue in many of the selected studies was the lack of detail on whether feature selection and hyperparameter optimisation was used and if so, what techniques. Only Fathima and Hundewale [41, Faisal et al. 72 and Veiga et al. 48 reported using a hyperparameter optimisation technique, the grid search, to find better model configurations. Others $445,49,51,56,72$ explicitly reported applying feature selection techniques to find the attributes that provided the best results for their models. Eight studies provide no detail at all $40,43,48,55$. Assuming these studies did not use these search and optimisation techniques, this represents an opportunity for further performance improvement.

Our review focused on studies that made use of clinical data in their ML and DL models. In some cases, this was not sole data source. For example, most of the selected studies used clinical data with other types of data, such as laboratory test results to support the diagnosis of a given arbovirus $40,42,43,45,48,51,55,56,72$, . Arboviral diseases are common in some of the poorest and remote regions of the world. Diagnosis based on laboratory tests requires both the availability of specialised equipment and staff to operate it. Even if available, in some instances this may add to the lapsed time and complexity of diagnosis. In contrast, a decision support tool based on clinical data using ML and DL is low-cost and rapid without the need of specialised resources.

In their guidelines for developing and reporting ML models in biomedical research, Luo et al. [89] suggest that the following evaluation metrics should be reported sensitivity, specificity, positive predictive value, negative predictive value, AUC, and calibration plot. Our analysis suggests a significant gap between the selected studies and these guidelines. While most studies evaluated sensitivity, specificity and predictive values, few studies offered comprehensive evaluation across all metrics. For example, only four studies measured performance using ROC and AUC.

\section{Conclusions}

This SLR presented an overview of the current literature that applies ML and DL models for the classification of arboviral diseases as a support for clinical diagnosis. Of the wide range of arboviruses, ML and DL research to diagnose arboviruses based on clinical data is limited to the three most common infections - Dengue, Chikungunya and Zika. We identified the main goals were largely binary classifications. In the case of 
Dengue, there is evidence of more nuanced attempts at multi-class classification e.g. Dengue severity and risk. Similarly, there is some evidence of differential diagnosis both within viruses (e.g. Dengue/Severe Dengue or Zika/Congenital Zika) however such studies are the exception. Although a limited sample, the majority of included studies focused on ML techniques rather than DL. Of the former, most were tree-based models (Decision Tree, Adaboost, Gradient Boost, Random Forest). The solitary DL model was a CNN, DenseNet. The most common evaluation metrics were accuracy, sensibility and specificity. Despite evidence of imbalanced datasets, only three included studies used AUC. In summary, ML and DL research to diagnose arboviruses is at a nascent level of maturity.

We suggest that having an efficient and comprehensive arboviral diseases clinical decision support system can improve the quality of the entire arboviral diseases clinical process thereby increasing the accuracy, precision and throughput of diagnosis (and mitigating the risk of misdiagnosis) and associated treatment. It would also help the physicians in their decision making process and, as a consequence, improving resource utilisation and patient quality of life as a whole. However, this requires a sustained, focused, and systematic approach to research that places differential diagnosis and reproducibility at its core. This implies greater coordination and sharing of data sets and greater detail regarding model configuration, feature selection, and hyper-parameter optimisation.

ML, and DL more specifically, have significant legal, ethical, and scientific limitations particularly with respect to healthcare decision making, not least the black box nature of many DL techniques. In terms of future research based on the results and open challenges of a Systematic Literature Review (SLR), we highlight the following directions, regarding the diagnosis and classification of arboviral diseases using ML and DL: (1) use of different data types and sources including clinical and demographic data, structured and unstructured data, for training and testing models; (2) applications of techniques to address imbalanced data; (3) greater exploration and evaluation of DL models and ensemble models, comprising ML and DL models, for arboviral classification; (4) greater focus on differential diagnosis within and across a wider range of arboviruses; (5) application of feature selection and hyperparameter optimisation techniques to fine-tune models; (6) consistent use of a more comprehensive set of evaluation metrics to accommodate imbalanced data, and (7) an easy-to-access diagnosis decision support system in remote regions allowing for intermittent connectivity.

\section{Acronyms}

ALP Alkaline phosphatase. 20

ALT Alanine transaminase. 20

AST Aspartate aminotransferase. 20

AUC Area Under the Curve. 10, 12, 21-25

BIA Bioelectrical Impedance Analysis. 15

BRBES Specialized Belief Rules System. 12

CART Classification and Regression Tree. 6, 10, 11, 13

CNN Convolutional Neural Network. 6, 9, 10, 23, 25

CZS Congenital Zika Syndrome. 3, 5, 13, 16 
DALYs disability-adjusted life years. 2

DBNs Deep Belief Networks. 23

DDM Dengue Diagnostic Model. 10

DF Dengue Fever. 5, 9, 11-13, 15-18

DHF Dengue Hemorrhagic Fever. 5, 9, 11-13, 15-18

DHF1 Dengue Hemorrhagic Fever 1. 5, 9

DHF2 Dengue Hemorrhagic Fever 2. 5, 9

DHF3 Dengue Hemorrhagic Fever 3. 5, 9

DL Deep Learning. 1, 3, 4, 6, 8, 9, 14, 23-25

DSNs Deep Stacking Networks. 23

DSPM Dengue Severity Prediction Model. 10

DSS Dengue Shock Syndrome. 5, 9, 11, 12, 15-17

ELISA Enzyme-linked immunoassay. 20

EOSBAS Eosinophile basofile count. 20

FLBES Fuzzy Logic Based Expert System. 12

FN False Negative. 21

FP False Positive. 21, 22

FPR False Positive Rate. 22

GRU Gated Recurrent Units. 23

HAI Hemagglutination-inhibition assay. 20

HGB Hemoglobin. 20

HTC Hematocrit. 11, 18, 20

ID3 Iterative Dichotomiser 3. 6

IgG Immunoglobulin G. 20

IgM Immunoglobulin M. 20

JEV Japanese encephalitis virus. 20

kNN K-Nearest Neighbors. 6, 8, 12, 13

LOO leave-one-out. 13, 16

LSTM Long Short-Term Memory. 23

LYMPH Lymphocite count. 20 
MCH Mean corpuscular hemoglobin. 20

MCHC Mean corpuscular hemoglobin concentration. 20

ML Machine Learning. 1, 3, 4, 6, 8, 9, 14, 23-25

MLP Multilayer Perceptron. 7, 8, 10, 11

MONO Monocyte count. 20

MPV Mean platelet volume. 20

NEUT Neutrophil count. 20

NN Neural Networks. 6, 8, 10, 12

NTDs Neglected tropical diseases. 1

OFI Other febrile illness. 15, 17

PDW Platelet distribuition width. 20

PLCR Platelet large cell ratio. 20

PLT Platelet count. 11, 18, 20

RBC Red bllod cells count. 20

RDW Red cell distribuition width. 20

RNNs Recurrent Neural Networks. 23

ROC Receiver Operating Characteristic. 21, 22, 24

RT-PCR Reverse transcription polymerase chain reaction. 14, 18, 20

SLR Systematic Literature Review. 1, 3, 7, 14, 16, 18, 21-25

SOM Self Organizing Map. 11, 15

SVM Support Vector Machine. 6, 7, 10-12

TN True Negative. 21, 22

TORCHS Toxoplasmosis, Rubella, Cytomegalovirus, Herpes Symplex, and Syphilis infections. 20

TP True Positive. 21, 22

TPR True Positive Rate. 22

WBC White blood ceels. 11, 18, 20 


\section{Acknowledgments}

VSS has a grant (062.00249/2020, EDITAL N. 006/2019 - UNIVERSAL AMAZONAS) from Fundação de Amparo à Pesquisa do Estado do Amazonas (FAPEAM)

(http://www.fapeam.am.gov.br/). The sponsor had no role in the study design, data collection and analysis, decision to publish, or preparation of the manuscript.

Authors would like to thank Conselho Nacional de Desenvolvimento Científico e Tecnológico (CNPq); Fundação de Amparo à Pesquisa do Estado do Amazonas (FAPEAM); Fundação de Vigilância em Saúde Dra. Rosemary Costa Pinto; Fundação de Amparo a Ciência e Tecnologia do Estado de Pernambuco (FACEPE); and Universidade de Pernambuco (UPE), an entity of the Government of the State of Pernambuco focused on the promotion of Teaching, Research and Extension.

\section{Appendix A}


Table 5. Overview of the primary studies.

\begin{tabular}{|c|c|c|c|c|c|c|c|c|}
\hline Primary studies & Year & Target classification & $\mathrm{ML}$ and/or DL & Model configuration & Software & Metrics & Hyperparameter optimisation & Feature selection \\
\hline Tanner et al. 40$]$ & 2008 & $\begin{array}{l}\text { (1) Dengue or not } \\
\text { (2) Severity of Dengue }\end{array}$ & Decision Tree & Not described & Not described & $\begin{array}{l}\text { Sensitivity, specificity } \\
\text { error rate, AUC }\end{array}$ & Not applied & Decision Tree \\
\hline Fathima and Hundewale 41] & 2012 & Dengue or not & Naive Bayes & Default values from the package e1071 & $\mathrm{R}$ & $\begin{array}{l}\text { Accuracy, sensitivity, } \\
\text { specificity, rate risk }\end{array}$ & Not applied & Not applied \\
\hline Sajana et al. 421 & 2018 & Dengue or not & $\begin{array}{l}\text { SVM } \\
\text { Decision Tree }\end{array}$ & $\begin{array}{l}\text { Not described } \\
\text { Not described }\end{array}$ & Not described & $\begin{array}{l}\text { Accuracy, precision, } \\
\text { recall, F-Measure }\end{array}$ & $\begin{array}{l}\text { Grid Search } \\
\text { Not applied }\end{array}$ & Not applied \\
\hline \multirow[t]{3}{*}{ Gambhir et al. 433} & 2018 & Dengue or not & $\begin{array}{l}\text { NN } \\
\text { NN }\end{array}$ & $\begin{array}{l}\text { Not described } \\
\text { hidden layers }=3 \\
\text { lr }=0.3 \\
\text { momentum }=0.25\end{array}$ & Not described & $\begin{array}{l}\text { Accuracy, sensitivity } \\
\text { specificity, error rate }\end{array}$ & Not applied & Not applied \\
\hline & & & Decision Tree & $\begin{array}{l}\text { criterion }=\text { Information Gain } \\
\text { size of split }=2 \\
\text { min leaf size }=2 \\
\text { min gain }=0.01 \\
\text { max depth }=20 \\
\text { confidence }=0.5\end{array}$ & & & & \\
\hline & & & Naive Bayes & $\begin{array}{l}\text { estimation method }=\text { greedy } \\
\text { min bandwidth }=0.01 \\
\text { num of kernels }=10\end{array}$ & & & & \\
\hline Sanjudevi and Savitha 44] & 2019 & Dengue or not & Decision Tree & Not described & WEKA & $\begin{array}{l}\text { Accuracy, sensitivity, } \\
\text { specificity, AUC }\end{array}$ & Not applied & Not applied \\
\hline \multirow[t]{2}{*}{ Ho et al. 45] } & 2020 & Dengue or not & $\begin{array}{l}\text { SVM } \\
\text { Decision Tree }\end{array}$ & $\begin{array}{l}\text { Not described } \\
\text { critierion }=\text { gini } \\
\text { min leaf size }=20 \\
\text { vval }=10 \\
\operatorname{cp}=0.01\end{array}$ & Not described & $\mathrm{ROC}, \mathrm{AUC}$ & Not applied & $\begin{array}{l}\text { Crude odds ratios } \\
\text { Adjusted odds ratios }\end{array}$ \\
\hline & & & $\begin{array}{l}\text { Logistic Regression } \\
\text { CNN }\end{array}$ & $\begin{array}{l}\text { solver }=\text { lbfgs } \\
\text { hidden lavers }=16\end{array}$ & & & & \\
\hline Potts et al. [55] & 2010 & Severity of Dengue & Decision Tree & $\begin{array}{l}\text { criterion = gini } \\
\text { min samples split }=0.05 \\
\text { max depth } 5 \\
\text { min impurity decrease }=0.0001\end{array}$ & SPSS Answer Tree 3.0 & Sensitivity, specificity & Not applied & Decision Tree \\
\hline Phakhounthong et al. [56] & 2018 & Severity of Dengue & Decision Tree & Not described & WEKA & $\begin{array}{l}\text { Accuracy, sensitivity, } \\
\text { specificity }\end{array}$ & Applied but not described & Logistic Regression \\
\hline Faisal et al. 72$]$ & 2010 & Risk of Dengue & $\mathrm{NN}$ & $\begin{array}{l}\text { neurons }=10 \\
\mathrm{lr}=0.1 \\
\text { momentum }=0.99 \\
\text { iterations }=20.000\end{array}$ & Not described & Accuracy & Grid Search & SOM \\
\hline Thitiprayoonwongse et al. [49] & 2012 & DF, DHF1, DHF2 or DHF3 & Decision Tree & (1) confidence $=0.4$ & Not described & $\begin{array}{l}\text { Accuracy, sensitivity, } \\
\text { specificity }\end{array}$ & Not applied & Decision Tree \\
\hline Arafiyah et al. 466$]$ & 2018 & DHF or not & $\begin{array}{l}\text { Random Forest } \\
\text { SVM }\end{array}$ & $\begin{array}{l}\text { (2) confidence }=0.3 \\
\text { (3) Not described } \\
\text { Not described } \\
\text { Not described }\end{array}$ & Orange & Accuracy, sensitivity & Not applied & Not applied \\
\hline \multirow[t]{7}{*}{ Fahmi et al. 50] } & 2020 & DF, DHF or DSS & $\begin{array}{l}\text { Naive Bayes } \\
\text { NN }\end{array}$ & $\begin{array}{l}\text { Not descrived } \\
\text { Not described } \\
\text { neurons }=100 \\
\text { activation }=\text { Relu } \\
\text { solver }=\text { Adam } \\
\text { reg alfa }=0.0001 \\
\text { iterations }=200\end{array}$ & Orange & $\begin{array}{l}\text { Accuracy, sensitivity, } \\
\text { precision }\end{array}$ & Not applied & ReliefF \\
\hline & & & Decision Tree & $\begin{array}{l}\text { criterion }=\text { Information gain } \\
\min \text { leaf }=5 \\
\text { min instances }=2 \\
\text { max deph }=100\end{array}$ & & & & \\
\hline & & & SVM & $\begin{array}{l}\mathrm{c}=100 \\
\text { kernel }=\mathrm{rbf} \\
\text { tolerance }=0.0010 \\
\text { max iteration }=100\end{array}$ & & & & \\
\hline & & & KNN & $\begin{array}{l}k=5 \\
\text { distance metric }=\text { euclidean } \\
\text { weight }=\text { uniform }\end{array}$ & & & & \\
\hline & & & Random Forest & $\begin{array}{l}\text { n estimators }=10 \\
\text { size of pplit }=5 \\
\text { criterion }=\text { gini }\end{array}$ & & & & \\
\hline & & & Naive Bayes & $\begin{array}{l}\text { Not described } \\
\text { Nomed }\end{array}$ & & & & \\
\hline & & & AdaBoost & $\begin{array}{l}\text { base estimator }=\text { Decision Tree } \\
\text { n estimators }=50 \\
\mathrm{l}=1.0 \\
\text { algorithm }=\text { SAMME.R } \\
\text { loss function }=\text { linear regression }\end{array}$ & & & & \\
\hline Hossain et al. 47$]$ & 2019 & Chikungunya or not & $\begin{array}{l}\text { Logistic Regression } \\
\text { NN }\end{array}$ & $\begin{array}{l}\text { Default configuration } \\
\text { Not described }\end{array}$ & Matlab & $\mathrm{AUC}$ & Not applied & Not applied \\
\hline \multirow[t]{4}{*}{ Veiga et al. $48 \mid$} & 2021 & Zika (CZS) or not & $\begin{array}{l}\text { SVM } \\
\text { Random Forest }\end{array}$ & $\begin{array}{l}\text { Not described } \\
\text { n estimators }=100 \\
\text { max depth }=5 \\
\text { size of split }=40\end{array}$ & & $\begin{array}{l}\text { Sensitivity, precision } \\
\text { F1-score }\end{array}$ & Grid search & Applied but not described \\
\hline & & & Gradient Boost & $\begin{array}{l}\text { max depth }=8 \\
\text { size of split }=5\end{array}$ & & & & \\
\hline & & & & Not described & & & & \\
\hline & & & Decision Tree & Not described & & & & \\
\hline Lee et al. 51] & 2012 & DF, DHF or Chikungunya & Decision Tree & $\begin{array}{l}\text { Not described } \\
\text { Binary recursive partitioning }\end{array}$ & $\mathrm{R}$ & $\begin{array}{l}\text { Sensitivity, specificity } \\
\text { AUC }\end{array}$ & Pruning & Logistic Regression \\
\hline
\end{tabular}




\section{References}

1. World Health Organization. Neglected Tropical Diseases; 2020.

2. Artsob H, Lindsay R, Drebot M. Arboviruses. In: Quah SR, editor. International Encyclopedia of Public Health (Second Edition). second edition ed. Oxford:

Academic Press; 2017. p. 154-160. Available from: https:

//www.sciencedirect.com/science/article/pii/B9780128036785000230

3. Lopes N, Nozawa C, Linhares REC. Características gerais e epidemiologia dos arbovírus emergentes no Brasil. Revista Pan-Amazônica de Saúde.

$2014 ; 5(3): 55-64$.

4. World Health Organization. Keeping the Vector Out: Housing improvements for vector control and sustainable development; 2017.

5. Paixão ES, Teixeira MG, Rodrigues LC. Zika, chikungunya and dengue: the causes and threats of new and re-emerging arboviral diseases. BMJ global health. 2018;3(Suppl 1):e000530.

6. Kalbus A, de Souza Sampaio V, Boenecke J, Reintjes R. Exploring the influence of deforestation on dengue fever incidence in the Brazilian Amazonas state. Plos one. 2021;16(1):e0242685.

7. LaDeau SL, Allan BF, Leisnham PT, Levy MZ. The ecological foundations of transmission potential and vector-borne disease in urban landscapes. Functional Ecology. 2015;29(7):889-901.

8. Esser HJ, Mögling R, Cleton NB, Van Der Jeugd H, Sprong H, Stroo A, et al. Risk factors associated with sustained circulation of six zoonotic arboviruses: a systematic review for selection of surveillance sites in non-endemic areas. Parasites \& vectors. 2019;12(1):265.

9. Girard M, Nelson CB, Picot V, Gubler DJ. Arboviruses: A global public health threat. Vaccine. 2020;38(24):3989-3994.

10. Lowe R, Lee S, Lana RM, Codeço CT, Castro MC, Pascual M. Emerging arboviruses in the urbanized Amazon rainforest. bmj. 2020;371.

11. Liu LE, Dehning M, Phipps A, Swienton RE, Harris CA, Klein KR. Clinical update on dengue, chikungunya, and Zika: what we know at the time of article submission. Disaster medicine and public health preparedness.

2017;11(3):290-299.

12. Cruz LCdTAd, Serra OP, Leal-Santos FA, Ribeiro ALM, Slhessarenko RD, Santos MAd. Natural transovarial transmission of dengue virus 4 in Aedes aegypti from Cuiabá, State of Mato Grosso, Brazil. Revista da Sociedade Brasileira de Medicina Tropical. 2015;48(1):18-25.

13. Heath CJ, Grossi-Soyster EN, Ndenga BA, Mutuku FM, Sahoo MK, Ngugi HN, et al. Evidence of transovarial transmission of Chikungunya and Dengue viruses in field-caught mosquitoes in Kenya. PLoS neglected tropical diseases. 2020;14(6):e0008362.

14. D'Ortenzio E, Matheron S, de Lamballerie X, Hubert B, Piorkowski G, Maquart M, et al. Evidence of sexual transmission of Zika virus. New England Journal of Medicine. 2016;374(22):2195-2198. 
15. Deckard DT, Chung WM, Brooks JT, Smith JC, Woldai S, Hennessey M, et al. Male-to-male sexual transmission of Zika virus-Texas, January 2016. Morbidity and Mortality Weekly Report. 2016;65(14):372-374.

16. Hoad VC, Speers DJ, Keller AJ, Dowse GK, Seed CR, Lindsay M, et al. First reported case of transfusion-transmitted Ross River virus infection. Med J Aust. 2015;202(5):267-9.

17. Zeng Z, Zhan J, Chen L, Chen H, Cheng S. Global, regional, and national dengue burden from 1990 to 2017: A systematic analysis based on the global burden of disease study 2017. EClinicalMedicine. 2021;32:100712.

18. Puntasecca CJ, King CH, LaBeaud AD. Measuring the global burden of chikungunya and Zika viruses: A systematic review. PLoS neglected tropical diseases. 2021;15(3):e0009055.

19. World Health Organization. Dengue and severe dengue; 2021.

20. World Health Organization. Zika virus; 2018.

21. Paniz-Mondolfi AE, Rodriguez-Morales AJ, Blohm G, Marquez M, Villamil-Gomez WE. ChikDenMaZika Syndrome: the challenge of diagnosing arboviral infections in the midst of concurrent epidemics; 2016.

22. Ioos S, Mallet HP, Goffart IL, Gauthier V, Cardoso T, Herida M. Current Zika virus epidemiology and recent epidemics. Medecine et maladies infectieuses. 2014;44(7):302-307.

23. Cerbino-Neto J, Mesquita EC, Amancio RT, Brasil PEAAd. Events preceding death among chikungunya virus infected patients: a systematic review. Revista da Sociedade Brasileira de Medicina Tropical. 2020;53.

24. Villamil-Gómez WE, González-Camargo O, Rodriguez-Ayubi J, Zapata-Serpa D, Rodriguez-Morales AJ. Dengue, chikungunya and Zika co-infection in a patient from Colombia. Journal of infection and public health. 2016;9(5):684-686.

25. Eligio-García L, Crisóstomo-Vázquez MDP, Caballero-García MdL, Soria-Guerrero M, Méndez-Galván JF, López-Cancino SA, et al. Co-infection of Dengue, Zika and Chikungunya in a group of pregnant women from Tuxtla Gutiérrez, Chiapas: Preliminary data. 2019. PLOS Neglected Tropical Diseases. 2020;14(12):e0008880.

26. Cavalcanti LPdG, Freitas ARR, Brasil P, Cunha RVd. Surveillance of deaths caused by arboviruses in Brazil: from dengue to chikungunya. Memorias do Instituto Oswaldo Cruz. 2017;112(8):583-585.

27. Maniero VC, Santos MO, Ribeiro RL, de Oliveira PA, da Silva TB, Moleri AB, et al. Dengue, chikungunya e zika vírus no brasil: situação epidemiológica, aspectos clínicos e medidas preventivas. Almanaque multidisciplinar de pesquisa. $2016 ; 1(1)$.

28. Marimoutou C, Vivier E, Oliver M, Boutin JP, Simon F. Morbidity and impaired quality of life 30 months after chikungunya infection: comparative cohort of infected and uninfected French military policemen in Reunion Island. Medicine. 2012;91(4):212-219. 
29. Rodriguez-Morales A, Cardona-Ospina J, Villamil-Gómez W, Paniz-Mondolfi A. How many patients with post-chikungunya chronic inflammatory rheumatism can we expect in the new endemic areas of Latin America? Rheumatology international. 2015;35(12):2091-2094.

30. Marques CDL, Duarte ALBP, Ranzolin A, Dantas AT, Cavalcanti NG, Gonçalves RSG, et al. Recommendations of the Brazilian Society of Rheumatology for diagnosis and treatment of Chikungunya fever. Part 1-Diagnosis and special situations. Revista brasileira de reumatologia. 2017;57:s421-s437.

31. World Health Organization. Chikungunya; 2020.

32. Zanluca C, Melo VCAd, Mosimann ALP, Santos GIVd, Santos CNDd, Luz K. First report of autochthonous transmission of Zika virus in Brazil. Memórias do Instituto Oswaldo Cruz. 2015;110(4):569-572.

33. Brasil P, Pereira Jr JP, Moreira ME, Ribeiro Nogueira RM, Damasceno L, Wakimoto M, et al. Zika virus infection in pregnant women in Rio de Janeiro. New England Journal of Medicine. 2016;375(24):2321-2334.

34. Costello A, Dua T, Duran P, Gülmezoglu M, Oladapo OT, Perea W, et al. Defining the syndrome associated with congenital Zika virus infection. Bulletin of the World Health Organization. 2016;94(6):406.

35. Driggers RW, Ho CY, Korhonen EM, Kuivanen S, Jääskeläinen AJ, Smura T, et al. Zika virus infection with prolonged maternal viremia and fetal brain abnormalities. New England Journal of Medicine. 2016;374(22):2142-2151.

36. Donalisio MR, Freitas ARR, Zuben APBV. Arboviruses emerging in Brazil: challenges for clinic and implications for public health. Revista de saude publica. 2017;51:30.

37. Dargan S, Kumar M, Ayyagari MR, Kumar G. A survey of deep learning and its applications: a new paradigm to machine learning. Archives of Computational Methods in Engineering. 2019; p. 1-22.

38. Chollet F. Deep learning with Python. Simon and Schuster; 2017.

39. Kitchenham B, Charters S. Guidelines for performing systematic literature reviews in software engineering. 2007;

40. Tanner L, Schreiber M, Low JG, Ong A, Tolfvenstam T, Lai YL, et al. Decision tree algorithms predict the diagnosis and outcome of dengue fever in the early phase of illness. PLoS Negl Trop Dis. 2008;2(3):e196.

41. Fathima SA, Hundewale N. Comparitive Analysis of Machine learning Techniques for classification of Arbovirus. In: Proceedings of 2012 IEEE-EMBS International Conference on Biomedical and Health Informatics. IEEE; 2012. p. 376-379.

42. Sajana T, Navya M, Gayathri Y, Reshma N. Classification of Dengue using Machine Learning Techniques. Int J Eng Technol. 2018;7(2.32):212-218.

43. Gambhir S, Malik SK, Kumar Y. The diagnosis of dengue disease: An evaluation of three machine learning approaches. International Journal of Healthcare Information Systems and Informatics (IJHISI). 2018;13(3):1-19.

44. Sanjudevi D, Savitha D. Dengue fever prediction using classification techniques. Int Res J Eng Technol (IRJET). 2019;6(02):558-563. 
45. Ho TS, Weng TC, Wang JD, Han HC, Cheng HC, Yang CC, et al. Comparing machine learning with case-control models to identify confirmed dengue cases. PLoS neglected tropical diseases. 2020;14(11):e0008843.

46. Arafiyah R, Hermin F, Kartika I, Alimuddin A, Saraswati I. Classification of Dengue Haemorrhagic Fever (DHF) using SVM, naive bayes and random forest. In: IOP Conference Series: Materials Science and Engineering. vol. 434. IOP Publishing; 2018. p. 012070.

47. Hossain MS, Sultana Z, Nahar L, Andersson K. An Intelligent System to Diagnose Chikungunya under Uncertainty. Journal of Wireless Mobile Networks, Ubiquitous Computing, and Dependable Applications. 2019;10(2):37-54.

48. Veiga RV, Schuler-Faccini L, França GV, Andrade RF, Teixeira MG, Costa LC, et al. Classification algorithm for congenital Zika Syndrome: characterizations, diagnosis and validation. Scientific Reports. 2021;11(1):1-7.

49. Thitiprayoonwongse D, Suriyaphol P, Soonthornphisaj N. Data mining of dengue infection using decision tree. Entropy. 2012;2:2.

50. Fahmi A, Purwitasari D, Sumpeno S, Purnomo MH. Performance Evaluation of Classifiers for Predicting Infection Cases of Dengue Virus Based on Clinical Diagnosis Criteria. In: 2020 International Electronics Symposium (IES). IEEE; 2020. p. 456-462.

51. Lee VJ, Chow A, Zheng X, Carrasco LR, Cook AR, Lye DC, et al. Simple clinical and laboratory predictors of Chikungunya versus dengue infections in adults. PLoS Negl Trop Dis. 2012;6(9):e1786.

52. Campesato O. Artificial Intelligence, Machine Learning, and Deep Learning. Stylus Publishing, LLC; 2020.

53. Esmaily H, Tayefi M, Doosti H, Ghayour-Mobarhan M, Nezami H, Amirabadizadeh A. A comparison between decision tree and random forest in determining the risk factors associated with type 2 diabetes. Journal of research in health sciences. 2018;18(2):412.

54. Singh S, Gupta P. Comparative study ID3, cart and C4. 5 decision tree algorithm: a survey. International Journal of Advanced Information Science and Technology (IJAIST). 2014;27(27):97-103.

55. Potts JA, Gibbons RV, Rothman AL, Srikiatkhachorn A, Thomas SJ, Supradish Po, et al. Prediction of dengue disease severity among pediatric Thai patients using early clinical laboratory indicators. PLoS Negl Trop Dis. 2010;4(8):e769.

56. Phakhounthong K, Chaovalit P, Jittamala P, Blacksell SD, Carter MJ, Turner P, et al. Predicting the severity of dengue fever in children on admission based on clinical features and laboratory indicators: application of classification tree analysis. BMC pediatrics. 2018;18(1):1-9.

57. Bonaccorso G. Machine learning algorithms. Packt Publishing Ltd; 2017.

58. Khoshgoftaar TM, Golawala M, Van Hulse J. An empirical study of learning from imbalanced data using random forest. In: 19th IEEE International Conference on Tools with Artificial Intelligence (ICTAI 2007). vol. 2. IEEE; 2007. p. 310-317. 
59. Ke G, Meng Q, Finley T, Wang T, Chen W, Ma W, et al. Lightgbm: A highly efficient gradient boosting decision tree. Advances in neural information processing systems. 2017;30:3146-3154.

60. Freund Y, Schapire RE, et al. Experiments with a new boosting algorithm. In: icml. vol. 96. Citeseer; 1996. p. 148-156.

61. Mayr A, Binder H, Gefeller O, Schmid M. The evolution of boosting algorithms. Methods of information in medicine. 2014;53(06):419-427.

62. Vapnik V. The nature of statistical learning theory. Springer science \& business media; 2013.

63. Haykin S. Redes neurais: princípios e prática. Bookman Editora; 2007.

64. Sharkawy AN. Principle of neural network and its main types. Journal of Advances in Applied \& Computational Mathematics. 2020;7:8-19.

65. Du KL, Swamy MN. Neural networks in a softcomputing framework. Springer Science \& Business Media; 2006.

66. Ahmad F, Isa NAM, Hussain Z, Osman MK. Intelligent medical disease diagnosis using improved hybrid genetic algorithm-multilayer perceptron network. Journal of medical systems. 2013;37(2):1-8.

67. Cybenko G. Approximation by superpositions of a sigmoidal function. Mathematics of control, signals and systems. 1989;2(4):303-314.

68. Paliwal M, Kumar UA. Neural networks and statistical techniques: A review of applications. Expert systems with applications. 2009;36(1):2-17.

69. Sahoo AK, Pradhan C, Das H. Performance evaluation of different machine learning methods and deep-learning based convolutional neural network for health decision making. In: Nature inspired computing for data science. Springer; 2020. p. 201-212.

70. Paterakis NG, Mocanu E, Gibescu M, Stappers B, van Alst W. Deep learning versus traditional machine learning methods for aggregated energy demand prediction. In: 2017 IEEE PES Innovative Smart Grid Technologies Conference Europe (ISGT-Europe). IEEE; 2017. p. 1-6.

71. Golovko V. Deep learning: an overview and main paradigms. Optical memory and neural networks. 2017;26(1):1-17.

72. Faisal T, Ibrahim F, Taib MN. A noninvasive intelligent approach for predicting the risk in dengue patients. Expert Systems with Applications. 2010;37(3):2175-2181.

73. Mitchell T. Machine Learning, McGraw-Hill Higher Education. New York. 1997;.

74. Ayer T, Chhatwal J, Alagoz O, Kahn Jr CE, Woods RW, Burnside ES. Comparison of logistic regression and artificial neural network models in breast cancer risk estimation. Radiographics. 2010;30(1):13-22.

75. Cover T, Hart P. Nearest neighbor pattern classification. IEEE transactions on information theory. 1967;13(1):21-27.

76. LeCun Y, Cortes C, Burges C. THE MNIST DATABASE: of handwritten digits. http://yannlecuncom/exdb/mnist/ Accessed in 25 Ago 2020. 1998;. 
77. London AJ. Artificial intelligence and black-box medical decisions: accuracy versus explainability. Hastings Center Report. 2019;49(1):15-21.

78. Huang G, Liu Z, Van Der Maaten L, Weinberger KQ. Densely connected convolutional networks. In: Proceedings of the IEEE conference on computer vision and pattern recognition; 2017. p. 4700-4708.

79. Zhu Y, Newsam S. Densenet for dense flow. In: 2017 IEEE international conference on image processing (ICIP). IEEE; 2017. p. 790-794.

80. Kononenko I. Estimating attributes: Analysis and extensions of RELIEF. In: European conference on machine learning. Springer; 1994. p. 171-182.

81. He H, Garcia EA. Learning from imbalanced data. IEEE Transactions on knowledge and data engineering. 2009;21(9):1263-1284.

82. Grandini M, Bagli E, Visani G. Metrics for Multi-Class Classification: an Overview. arXiv preprint arXiv:200805756. 2020;.

83. Zhe Hui Hoo DT Jane Candlish. What is an ROC curve? Emergency Medicine Journal. 2017;34:3

84. Xiao C, Choi E, Sun J. Opportunities and challenges in developing deep learning models using electronic health records data: a systematic review. Journal of the American Medical Informatics Association. 2018;25(10):1419-1428.

85. Watson DS, Krutzinna J, Bruce IN, Griffiths CE, McInnes IB, Barnes MR, et al. Clinical applications of machine learning algorithms: beyond the black box. Bmj. $2019 ; 364$.

86. Rudin C. Stop explaining black box machine learning models for high stakes decisions and use interpretable models instead. Nature Machine Intelligence. $2019 ; 1(5): 206-215$.

87. Castelvecchi D. Can we open the black box of AI? Nature News. 2016;538(7623):20.

88. Pawar U, O'Shea D, Rea S, O'Reilly R. Explainable ai in healthcare. In: 2020 International Conference on Cyber Situational Awareness, Data Analytics and Assessment (CyberSA). IEEE; 2020. p. 1-2.

89. Luo W, Phung D, Tran T, Gupta S, Rana S, Karmakar C, et al. Guidelines for developing and reporting machine learning predictive models in biomedical research: a multidisciplinary view. Journal of medical Internet research. 2016;18(12):e323. 